\title{
A Novel Generalization of Trigonometric Bézier Curve and Surface with Shape Parameters and Its Applications
}

\author{
Sidra Maqsood, ${ }^{1}$ Muhammad Abbas ${ }^{(D)},{ }^{1}$ Gang Hu, ${ }^{2}$ Ahmad Lutfi Amri Ramli, ${ }^{3}$ \\ and Kenjiro T. Miura ${ }^{4}$ \\ ${ }^{1}$ Department of Mathematics, University of Sargodha, 40100 Sargodha, Pakistan \\ ${ }^{2}$ Department of Applied Mathematics, Xi'an University of Technology, 710054 Xi'an, China \\ ${ }^{3}$ School of Mathematical Sciences, Universiti Sains Malaysia, George Town, 11800 Penang, Malaysia \\ ${ }^{4}$ Department of Mechanical Engineering, Shizuoka University, Shizuoka, Japan \\ Correspondence should be addressed to Muhammad Abbas; muhammad.abbas@uos.edu.pk
}

Received 11 October 2019; Revised 21 March 2020; Accepted 15 April 2020; Published 25 May 2020

Academic Editor: Ruben Sevilla

Copyright (c) 2020 Sidra Maqsood et al. This is an open access article distributed under the Creative Commons Attribution License, which permits unrestricted use, distribution, and reproduction in any medium, provided the original work is properly cited.

\begin{abstract}
Adopting a recurrence technique, generalized trigonometric basis (or GT-basis, for short) functions along with two shape parameters are formulated in this paper. These basis functions carry a lot of geometric features of classical Bernstein basis functions and maintain the shape of the curve and surface as well. The generalized trigonometric Bézier (or GT-Bézier, for short) curves and surfaces are defined on these basis functions and also analyze their geometric properties which are analogous to classical Bézier curves and surfaces. This analysis shows that the existence of shape parameters brings a convenience to adjust the shape of the curve and surface by simply modifying their values. These GT-Bézier curves meet the conditions required for parametric continuity $\left(C^{0}, C^{1}, C^{2}\right.$, and $\left.C^{3}\right)$ as well as for geometric continuity $\left(G^{0}, G^{1}\right.$, and $\left.G^{2}\right)$. Furthermore, some curve and surface design applications have been discussed. The demonstrating examples clarify that the new curves and surfaces provide a flexible approach and mathematical sketch of Bézier curves and surfaces which make them a treasured way for the project of curve and surface modeling.
\end{abstract}

\section{Introduction}

The study of curves and surfaces plays a very significant role in computer-aided geometric design (CAGD) and computer graphics (CG). CAGD deals with the composition and representation of free-form curves and surfaces. Numerous applications which demand free-form curves and surfaces arise in science and engineering, e.g., car bodies, ship hulls, airplane, and propeller blades. The use and effectiveness of curves in modeling are resolute by the type of input data and their consequence on the control of the resulting curve. Curves representation, related to the control points, flexible enough to bend and twist or change the curve shape by changing one or more control points as the designers' requirements are big challenges in curve modelling. For these reasons, parametric representation of curves and surfaces is mostly used to handle these challenges.

Bézier curves and surfaces have been extensively used in CG and CAGD because of their valuable properties. Bézier curves are parametric curves and are constructed by using Bernstein polynomials as basis functions. To change the shape of the classical Bézier curves, their control points are essential to be adjusted because they have no shape parameters. In CAD/CAM technology fields, creating more suitable skills of scheming and amending of Bézier curve is an important research subject. Since traditional Bézier curves can be obtained by control points and Bernstein basis functions, after creating Bézier curves and 
surfaces, we can construct different shapes by using parametric and geometric continuities which fulfill our design requirements. Since shape designing is a timeconsuming process, usually we cannot execute our required design in one step even though by using continuity conditions, especially, when, we are going to establish some complex curves and surfaces by the help of those Bézier curves. In order to overcome this cumbersome problem, we construct GT-Bézier curves/surfaces based on GT-basis functions with two shape parameters instead of any bunch of parameters [1]. Since trigonometric Bézier curve with the shape parameters is more continuous as compared to polynomial Bézier curve, by the variation of shape parameters, we can modify the shape according to our own choice.

In practical applications, the appearance design of many products is relatively complex and cannot be illustrated by a single curve/surface. Therefore, there is a necessity to model such products using adjacent curves/ surfaces with some continuities. The authors in [2] derived the $G^{1}, G^{2}$ geometric continuity conditions only for H-Bézier curves of degree $n$. Hu et al. [3-5] also derived the continuity conditions between generalized Bézier-like surfaces with multiple shape parameters in order to resolve the issue of shape design for complex surfaces in engineering. In industrial applications, computer-aided machines have the ability to cut the shapes like circles or helices or the shapes of many objects designed with the help of straight lines and circle arcs. Therefore, it is useful to have curves piecewisely spanned by linear polynomials, sine and cosine, i.e., cycloidal splines (also called helix splines) to approximate such objects [6].

Many authors have constructed numerous varieties of Bézier curve/surface design. A class of extension of Bézier curve is constructed by Han and Liu [7], Wu et al. [8, 9], and Liu [10]. Due to the presence of one shape control parameter in all the above cited work, the control on the shape of Bézier curve has made more desirable, but still it is limited. Han et al. [11] suggested shape modification of cubic quasi-Bézier curve to enhance the shape adjustability of the curves. In [12], Qin et al. described the extension of cubic Bézier curve with different shape parameters and also its continuity conditions and applications. An extension of quartic Bézier curve with three shape parameters is presented by Zhu et al. [13], which is a continuation to a forth degree Bézier curve with a single parameter which improved the shape control of the curve. In [14], an extension of quartic Bézier curve is presented by Zhang et al. which not only inherits the outstanding properties of quadric Bézier curve but also fits the control polygon. Graphical examples with valuable design of curves and surfaces are also given in this literature. Yan and Liang [15] presented an extension of the Bézier model with all the properties. The newly created curves and surfaces by Qin et al. [16] not only have most properties of the corresponding classical Bézier curves and surfaces of order $n$ but they also help to modify the shapes by using various shape parameters. Hu et al. [17, 18] described the rotation surfaces by using polynomial basis and shape modification of various curves by using shape parameters. The generalized B-spline (GB-spline) functions of arbitrary order having all the basic properties of the curve are presented by Ksasov and Sattayatham in [19]. Zhang and Krause [20] presented the unified trigonometric basis and the hyperbolic basis with a shape parameter. The functional B-splines (FB-splines) and subdivision $\mathrm{B}$-splines (SB-splines) are also presented with a geometric proof of curvature continuity for SB-splines. Lü et al. [21] constructed the trigonometric polynomial B-spline curves in which they have many similar properties to traditional B-splines. Based on the explicit representation of the curves, the subdivision formulae for this new kind of curve are also presented in this literature. Wang et al. [22] described the subdivision formulae of the new kind of NAUT B-spline curves. The generation of tensor product surfaces by these new splines and unified and extended form of three types of splines are also studied in this work.

In $[23,24]$, Han presented the cubic and quadratic trigonometric polynomial curves with the shape parameters. The author used them for various designing purposes with the help of continuity conditions. Nikolis and Seimenis [25] presented the special nonlinear dynamical systems by using cubic trigonometric splines. The existence of the unique spline approximation is proved, and the convergence order of the method is shown to be cubic. The methods based on various spline techniques for planning and fast modifications of a trajectory for robot manipulators are investigated by Dyllong and Visioli in [26]. Su and Zou [27] designed the manipulator trajectory using algebraic-trigonometric Hermite polynomial curves and interpolated the data points for the manipulator of these curves.

Schweikert in [28] presented the use of a linearized mathematical spline for interpolation between given points which occasionally yields extraneous inflection points for some applications. Mazure [29] defined the Chebyshev-Bernstein basis as the dual bases of the linear functional giving the control points in which they shared the same properties as the Bernstein bases in polynomial spaces. Xu and Wang [30] presented the two unified mathematic models of conics and polynomial curves by AHT Bézier curves and NUAHT B-spline curves. Zhu and Liu [31] presented a class of trigonometric Bernstein-type basis functions with four different shape parameters. The continuity conditions and various modeling are also presented in this work. Mainer et al. [32] discussed several alternatives to the rational Bézier model based on using curves generated by mixing polynomial and trigonometric functions and expressing them in bases with optimal shape 
preserving properties (normalized B-bases). In [33], Wang and Fang unified and extended the polynomial, trigonometric, and hyperbolic splines by a new kind of UE-spline over a space.

Bosner and Rogina in [34] proposed numerically stable algorithms for cycloidal splines. They developed a corner cutting algorithm for lower-order cycloidal curves through a straightforward generalization. Costantini et al. [35] presented the approximation power, the existence of a normalized B-basis, and the structure of a degree-raising process for spaces of the form requiring suitable assumptions on the functions. Mainer and Peña [36] presented the quadratic cycloidal curves associated to equally spaced knots and the properties of the generated curves for a specific domain. Gang et al. [37] constructed a unified approach, the generalized nonuniform B-splines, and studied the corresponding isogeometric analysis framework for solving the partial differential equation. The proposed frameworks have several advantages such as high accuracy and easy-to-compute derivatives and integrals due to the nonrational form when compared with the NURBS-IGA method.

This paper defines the work based on the construction of new GT-basis functions of order $n(n>2)$ which are described by taking a set of basis functions of degree 2 with two shape parameters, with identical characteristics to the classical Bernstein basis functions. As an alternative technique of representing curves/surfaces, GT-Bézier curves/surfaces not only demonstrate the valuable characteristics of Bézier curves/surfaces but also allow efficient shape modification by altering the values of shape parameters. In order to resolve the problem of not being able to construct complex curves/surfaces using a single curve/ surface, we study the parametric and geometric continuity conditions for GT-Bézier curves/surfaces of degree $n$. The continuity conditions of $C^{3}$ and $G^{2}$ between two adjacent GT-Bézier curves and surfaces are proposed using terminal properties of GT-Bézier curves/surfaces of degree $n$. The present GT-basis functions and GT-Bézier curves/surfaces of degree $n$ are novel for the smooth connection between two adjacent GT-Bézier curves by $C^{3}$ continuity conditions and as far as we are aware, it has never been employed for thispurpose before.

This paper is laid out as follows: in Section 2, generalized trigonometric basis functions are constructed, and their properties are discussed. The GT-Bézier curves and surfaces are proposed with their properties in Sections 3 and 7, respectively. The geometric implication of the shape parameters will be examined in Section 4. Sections 5 and 6 provide the continuity conditions, some design applications, and examples of GT-Bézier curves and surfaces. A summarized conclusion is given in Section 8 .

\section{GT-Basis Function}

We construct the GT-basis functions by using a recursive relation in this section.

Definition 1. For $(-1 \leq \alpha, \beta \leq 1)$ and $(0 \leq z \leq 1)$, the functions

$$
\left\{\begin{array}{l}
w_{0,2}(z)=\left(1-\sin \left(\frac{\pi}{2} z\right)\right)\left(1-\alpha \sin \left(\frac{\pi}{2} z\right)\right), \\
w_{1,2}(z)=\left(1-w_{0,2}(z)-w_{2,2}(z)\right), \\
w_{2,2}(z)=\left(1-\cos \left(\frac{\pi}{2} z\right)\right)\left(1-\beta \cos \left(\frac{\pi}{2} z\right)\right),
\end{array}\right.
$$

are known as GT-basis functions of degree 2. The function $w_{i, m}(z)(i=0,1, \ldots, m)$ described recursively for any integer $m(m \geq 3)$ as

$$
w_{i, m}(z)=\left(1-\sin \left(\frac{\pi}{2} z\right)\right) w_{i, m-1}(z)+\sin \left(\frac{\pi}{2} z\right) w_{i-1, m-1}(z)
$$

is GT-basis function of the $m$ th order. In situation, when $i=-1$ or $i>m$, the function $w_{i, m}(z)=0$.

2.1. Properties of GT-Basis Functions. The GT-basis functions enjoy many properties as follows:

(1) Partition of unity:

$$
\sum_{i=1}^{m} w_{i, m}(z)=1
$$

(2) Nonnegativity: for $\alpha, \beta \in[-1,1], \quad w_{i, m}(z) \geq 0$ (i= $0,1, \ldots, m)$.

(3) Terminal property: $\forall i=0,1,2,3, \ldots, m(m \geq 2)$, $w_{0, m}(0)=1, w_{i, m}(0)=0(i=1,2, \ldots, m), w_{i, m}(1)=$ $0(i=0,1, \ldots, m-1)$, and $w_{m, m}(1)=1$.

(4) Derivative at the corner points: 


$$
\begin{aligned}
& w_{i, m}^{\prime}(0)= \begin{cases}-\frac{\pi}{2}\left(m_{1}+\alpha\right), & i=0, \\
\frac{\pi}{2}\left(m_{1}+\alpha\right), & i=1, \\
0, & \text { other, }\end{cases} \\
& w_{i, m}^{\prime}(1)= \begin{cases}-\frac{\pi}{2}(1+\beta), & i=m-1, \\
\frac{\pi}{2}(1+\beta), & i=m, \\
0, & \text { other, }\end{cases} \\
& w_{i, m}^{\prime \prime}(0)= \begin{cases}\frac{\pi^{2}}{4}\left(m_{1} m_{2}+2\left(m_{2}+1\right) \alpha\right), \\
-\frac{\pi^{2}}{4}\left(2 m_{1} m_{2}+2\left(2 m_{2}+1\right) \alpha+(1-\beta)\right), & i=1, \\
\frac{\pi^{2}}{4}\left(m_{1} m_{2}+2 m_{2} \alpha+(1-\beta)\right), & i=0, \\
0, & \text { other, }\end{cases}
\end{aligned}
$$$$
w_{i, m}^{\prime \prime}(1)= \begin{cases}\frac{-\pi^{2}}{4}(\alpha-1), & i=m-2, \\ \frac{\pi^{2}}{4}\left(m_{2}-2 \beta+(\alpha-1)\right), & i=m-1, \\ -\frac{\pi^{2}}{4}\left(m_{2}-2 \beta\right), & i=m, \\ 0, & \text { other, }\end{cases}
$$

$$
w_{i, m}^{\prime \prime}(0)= \begin{cases}\frac{-\pi^{3}}{8}\left[m_{3}^{3}-2 m_{2}-1+\left(3 m_{1} m_{2}-1\right) \alpha\right], & i=0, \\ \frac{\pi^{3}}{8}\left[m_{3}^{3}-2 m_{2}+2 m_{1} m_{2} m_{3}-1+\left(3 m_{1} m_{2}+6 m_{2}^{2}-1\right) \alpha-3 m_{2}(1-\beta)\right], & i=1, \\ \frac{-\pi^{3}}{8}\left[3 m_{1} m_{2} m_{3}+m_{2}\left(3 m_{2}-1\right) \alpha+6 m_{2}(1-\beta)\right], & i=2, \\ \frac{\pi^{3}}{8}\left[m_{1} m_{2} m_{3}+3 m_{2} m_{3} \alpha+3 m_{2}(1-\beta)\right], & i=3, \\ 0, & \text { other }\end{cases}
$$

$$
w_{i, m}^{\prime}(1)= \begin{cases}\frac{-\pi^{3}}{8}\left[\left(3 m_{2}+1\right)(1+\beta)\right], & i=m, \\ \frac{\pi^{3}}{8}\left[\left(6 m_{2}+1\right)(1+\beta)\right], & i=m-1, \\ \frac{-\pi^{3}}{8}\left[3 m_{2}(1+\beta)\right], & i=m-2, \\ 0, & \text { other }\end{cases}
$$


where $m_{k}=(m-k)(k=1,2,3)$.

Using Definition 3.1, the GT-basis functions for $m=3,4$, and 5 can be defined as follows:

(1) For $m=3$, we have

$$
\left\{\begin{array}{l}
w_{0,3}(z)=\left(1-\sin \left(\frac{\pi}{2} z\right)\right) w_{0,2}(z), \\
w_{1,3}(z)=\sin \left(\frac{\pi}{2} z\right) w_{0,2}(z)+\left(1-\sin \left(\frac{\pi}{2} z\right)\right) w_{1,2}(z), \\
w_{2,3}(z)=\left(1-\sin \left(\frac{\pi}{2} z\right)\right) w_{2,2}(z)+\sin \left(\frac{\pi}{2} z\right) w_{1,2}(z), \\
w_{3,3}(z)=\sin \left(\frac{\pi}{2} z\right) w_{2,2}(z) .
\end{array}\right.
$$

Figure 1(a) exhibits the curves generated by cubic GT-basis functions for $\alpha, \beta=-1$ (blue dotted), -0.5 (green), 0.5 (red), and 1 (black dashed).

(2) For $m=4$, we have

$$
\left\{\begin{array}{l}
w_{0,4}(z)=\left(1-\sin \left(\frac{\pi}{2} z\right)\right)^{2} w_{0,2}(z), \\
w_{1,4}(z)=\left(1-\sin \left(\frac{\pi}{2} z\right)\right)\left(2 \sin \left(\frac{\pi}{2} z\right) w_{0,2}(z)+\left(1-\sin \left(\frac{\pi}{2} z\right)\right) w_{1,2}(z)\right), \\
w_{2,4}(z)=\sin \left(\frac{\pi}{2} z\right)^{2} w_{0,2}(z)+\left(1-\sin \left(\frac{\pi}{2} z\right)\right)^{2} w_{2,2}(z)+2 \sin \left(\frac{\pi}{2} z\right)\left(1-\sin \left(\frac{\pi}{2} z\right)\right) w_{1,2}(z), \\
w_{3,4}(z)=\sin \left(\frac{\pi}{2} z\right)\left(2\left(1-\sin \left(\frac{\pi}{2} z\right)\right) w_{2,2}(z)\right)+\sin \left(\frac{\pi}{2} z\right) w_{1,2}(z), \\
w_{4,4}(z)=\sin \left(\frac{\pi}{2} z\right)^{2} w_{2,2}(z) .
\end{array}\right.
$$

Figure 1(b) depicts the graphs of quartic GT-basis functions with $\alpha, \beta=-1$ (blue), -0.5 (red dotted), 0.5 (orange), and 1 (black dashed).
(3) For $m=5$, we have

$$
\left\{\begin{array}{l}
w_{0,5}(z)=\left(1-\sin \left(\frac{\pi}{2} z\right)\right)^{3} w_{0,2}(z), \\
w_{1,5}(z)=\left(1-\sin \left(\frac{\pi}{2} z\right)\right)^{2}\left(3 \quad \sin \left(\frac{\pi}{2} z\right) w_{0,2}(z)+\left(1-\sin \left(\frac{\pi}{2} z\right)\right) w_{1,2}(z),\right. \\
w_{2,5}(z)=\left(1-\sin \left(\frac{\pi}{2} z\right)\right)\left(3 \quad \sin \quad\left(\frac{\pi}{2} z\right)^{2} w_{0,2}(z)+3 \quad \sin \left(\frac{\pi}{2} z\right)\left(1-\sin \left(\frac{\pi}{2} z\right)\right) w_{1,2}(z)+\left(1-\sin \left(\frac{\pi}{2} z\right)\right)^{2} w_{2,2}(z),\right. \\
w_{3,5}(z)=\sin \left(\frac{\pi}{2} z\right)\left(\sin \quad\left(\frac{\pi}{2} z\right)^{2} w_{0,2}(z)+3 \quad \sin \left(\frac{\pi}{2} z\right)\left(1-\sin \left(\frac{\pi}{2} z\right)\right) w_{1,2}(z)+3\left(1-\sin \left(\frac{\pi}{2} z\right)\right)^{2} w_{2,2}(z),\right. \\
w_{4,5}(z)=\sin \left(\frac{\pi}{2} z\right)^{2}\left(3\left(1-\sin \left(\frac{\pi}{2} z\right)\right) w_{2,2}(z)+\sin \left(\frac{\pi}{2} z\right) w_{1,2}(z),\right. \\
w_{5,5}(z)=\sin \left(\frac{\pi}{2} z\right)^{3} w_{2,2}(z) .
\end{array}\right.
$$


The graphs of 5th and 10th degree GT-basis functions with shape parameters $\alpha, \beta=-1$ (blue dotted), -0.3 (red), 0.5 (green), and 1 (black dashed) are given in Figures 1(c) and $1(\mathrm{~d})$, respectively.

\section{Construction of GT-Bézier Curves}

Definition 2. For any given control points $P_{i} \in R^{2}$ or $R^{3}(i=$ $0,1, \ldots, m)$ and shape parameters $\alpha, \beta$, the GT-Bézier curve can be constructed as

$$
S(z)=\sum_{i=0}^{m} P_{i} w_{i, m}(z)
$$

where $w_{i, m}(z)$ are GT-basis functions (2).
3.1. Properties of GT-Bézier Curves. In this section, we examine the geometric properties of GT-Bézier curves which are identical to classical Bézier curves.

(1) Terminal properties:

$$
\left\{\begin{array}{l}
S(0)=P_{0}, \\
S(1)=P_{m}, \\
S^{\prime}(0)=\frac{\pi}{2}\left(m_{1}+\alpha\right)\left(P_{1}-P_{0}\right), \\
S^{\prime}(1)=\frac{\pi}{2}(1+\beta)\left(P_{m}-P_{m-1}\right) .
\end{array}\right.
$$

If $m=3$,

$$
\left\{\begin{array}{l}
S^{\prime \prime}(0)=\frac{\pi^{2}}{4}\left[2(1+2 \alpha) P_{0}-(5+6 \alpha-\beta) P_{1}+(3+2 \alpha-\beta) P_{2}\right], \\
S^{\prime \prime}(1)=\frac{\pi^{2}}{4}\left[(1-\alpha) P_{1}+(\alpha-2 \beta) P_{2}-(1-2 \beta) P_{3}\right], \\
S^{\prime}(0)=\frac{\pi^{3}}{8}\left[(2-5 \alpha) P_{0}+(1+11 \alpha-3 \beta) P_{1}-3(2+2 \alpha-2 \beta) P_{2}+(3-3 \beta) P_{3}\right], \\
S^{\prime}(1)=\frac{\pi^{3}}{8}(1+\beta)\left[7 P_{2}-3 P_{1}-4 P_{3}\right] .
\end{array}\right.
$$

If $m \geq 3$,

$$
\left\{\begin{array}{l}
S^{\prime \prime}(0)=\frac{\pi^{2}}{4}\left[\left(m_{1} m_{2}+2 m_{2} \alpha\right)\left(P_{2}-2 P_{1}+P_{0}\right)+(1-\beta)\left(P_{2}-P_{1}\right)-2 \alpha\left(P_{1}-P_{0}\right)\right], \\
S^{\prime \prime}(1)=\frac{\pi^{2}}{4}\left[(\alpha-1)\left(P_{m-1}-P_{m-2}\right)-\left(m_{2}-2 \beta\right)\left(P_{m}-P_{m-1}\right)\right], \\
S^{\prime \prime}(0)=\frac{\pi^{3}}{8}\left[\left(m_{3}^{3}-2 m_{2}-1+\left(3 m_{1} m_{2}-1\right) \alpha\right)\left(P_{1}-P_{0}\right)+3 m_{2}(1-\beta)\left(P_{3}-2 P_{2}+P_{1}\right)+m_{1} m_{2} m_{3}\left(P_{3}-3 P_{2}+2 P_{1}\right)\right. \\
\left.\quad+m_{2} \alpha\left(3 m_{3} P_{3}-\left(3 m_{2}-1\right) P_{2}+6 m_{2} P_{1}\right)\right), \\
S^{\prime \prime}(1)=\frac{\pi^{3}}{8}\left[(1+\beta)\left(P_{m-1}-P_{m-2}\right)-3 m_{2}(1+\beta)\left(P_{m}-2 P_{m-1}+P_{m-2}\right)\right] .
\end{array}\right.
$$

(2) Convex hull property: the whole GT-Bézier curve segment absolutely lies inward its control polygon.

(3) Geometric invariance: the shape of the GT-Bézier curve does not depend on the choice of the chosen coordinate. This property can be visualized by Figure 2. Figure 2(a) shows that black is the first curve $S(z)$ with control points $P_{i}$, and the blue curve is obtained by adding a point $O^{\prime}=(3,9)$ in $S(z)$. 


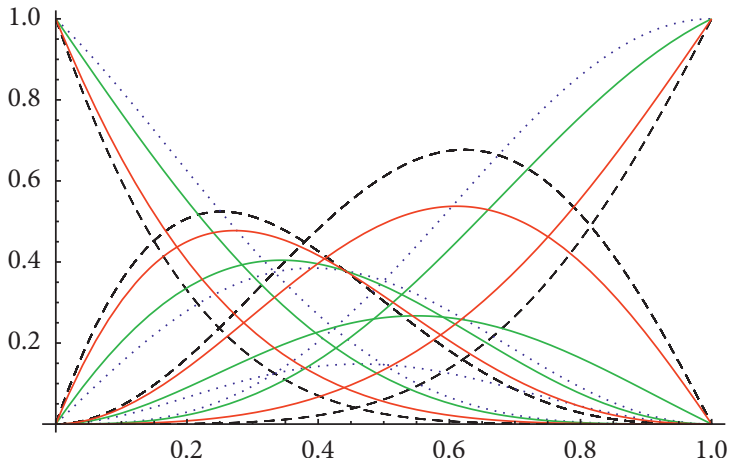

(a)

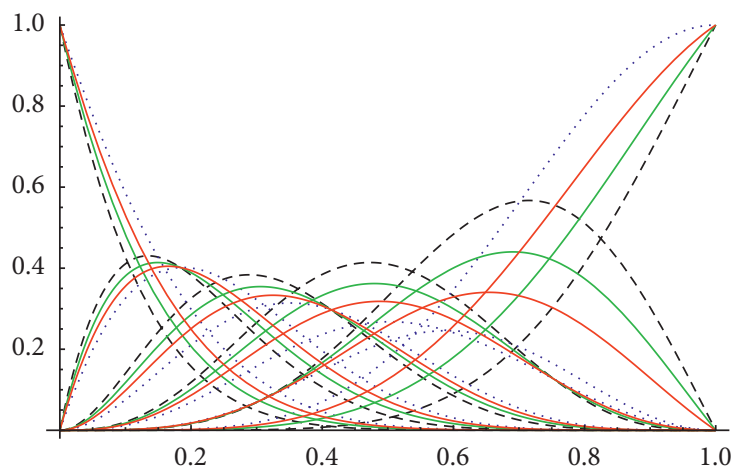

(c)

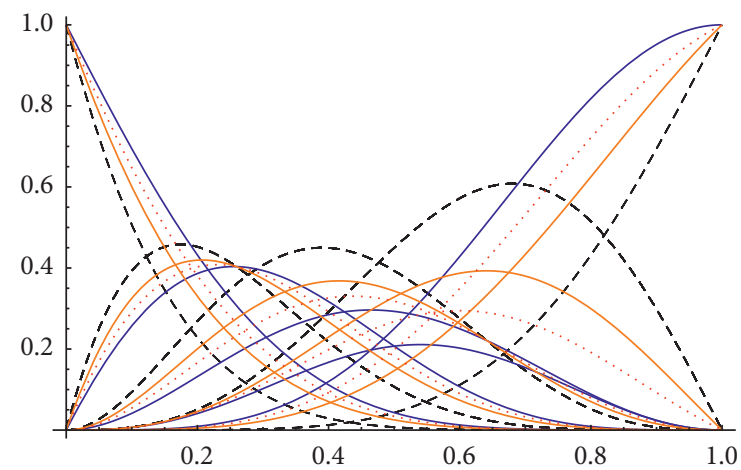

(b)

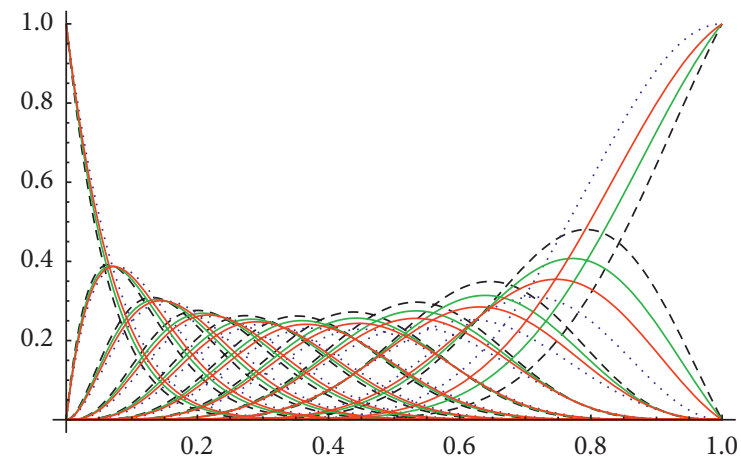

(d)

Figure 1: GT-basis functions of multiple degrees with multiple shape parameters. (a) $m=3$. (b) $m=4$. (c) $m=5$. (d) $m=10$.

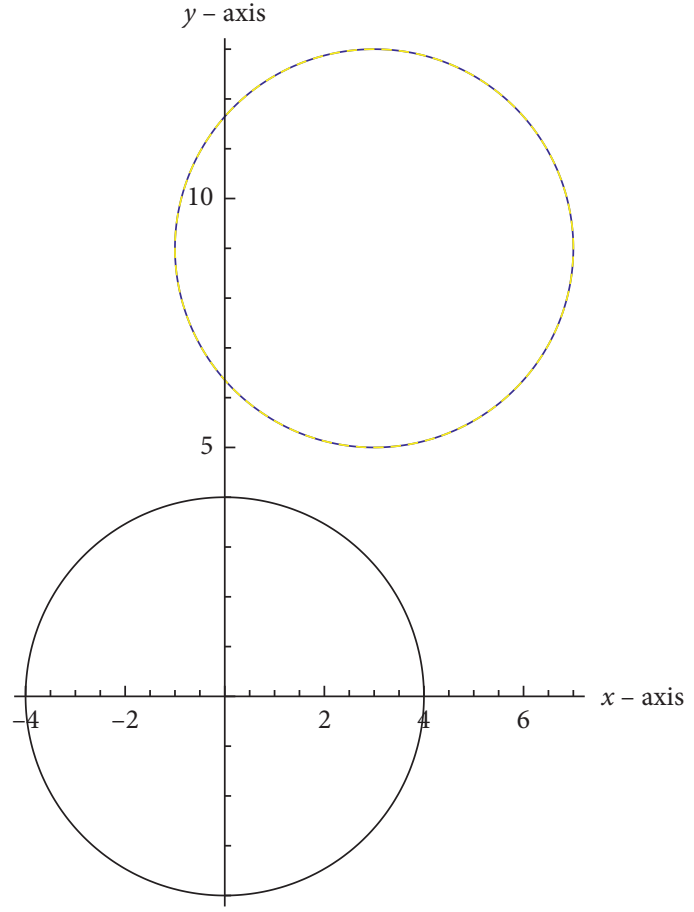

(a)

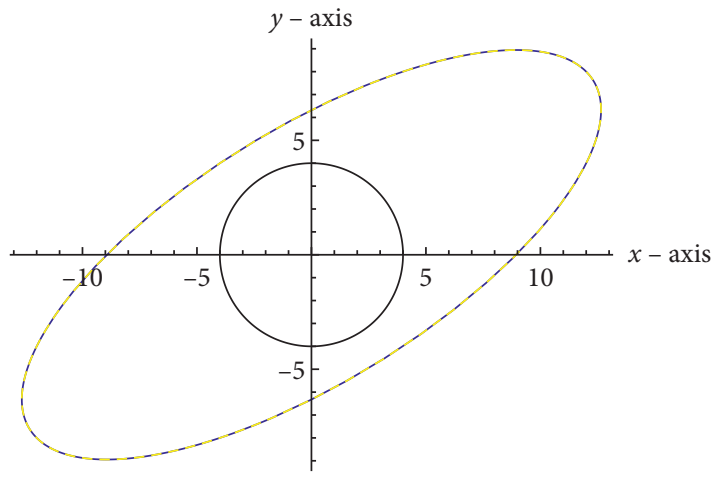

(b)

Figure 2: Geometric invariance of GT-Bézier curves. (a) Geometric invariance with respect to addition. (b) Geometric invariance with respect to multiplication. 
Yellow-dotted circle on the blue circle is obtained by the control points $P_{i}+O^{\prime}$. Similarly, Figure 2(b) shows that black is the first curve $S(z)$ with control points $P_{i}$, the blue curve is represented by the control points $P_{i} * Y^{\prime}$, and the yellow-dotted curve printed on the blue curve is obtained by multiplying $S(z)$ with a matrix $Y^{\prime}=\left(\begin{array}{ll}1 & 2 \\ 3 & 1\end{array}\right)$.

\section{Geometric Implication of Shape Parameters}

4.1. Correlation amid Shape Parameters and Stationary Points on the Curves. From the definition of GT-Bézier curve (8), we confirm that it is a linear function for every shape parameter, and

$$
\begin{aligned}
& \frac{\partial S(z)}{\partial \alpha}=\left(\begin{array}{c}
m-2 \\
i
\end{array}\right)\left(1-\sin \left(\frac{\pi}{2} z\right)\right)^{m-1-i}\left(\sin \left(\frac{\pi}{2} z\right)\right)^{i+1} \\
&\left(P_{i+1}-P_{i}\right), \quad i=0,1, \ldots, m-2, \\
& \frac{\partial S(z)}{\partial \beta}=\left(\begin{array}{c}
m-2 \\
i-1
\end{array}\right) \cos \left(\frac{\pi}{2} z\right)\left(1-\cos \left(\frac{\pi}{2} z\right)\right) \\
&\left(1-\sin \left(\frac{\pi}{2} z\right)\right)^{m-1-i}\left(\sin \left(\frac{\pi}{2} z\right)\right)^{i-1}\left(P_{i}-P_{i+1}\right) \\
& i=1,2, \ldots, m-1 .
\end{aligned}
$$

Therefore, there is no relationship among $(\partial S(z) / \partial \alpha)$ and $\alpha$ and $(\partial S(z) / \partial \beta)$ and $\beta$. Modifying one shape parameter $\alpha$ or $\beta$, the point $S(z)$ on the curve changed linearly for an unmovable control polygon and defined value of $z$. The route modification is as follows:

$$
\begin{cases}P_{i} P_{i+1}, & i=0,1, \ldots, m-2 \text { for } \alpha \\ P_{i+1} P_{i}, & i=1,2, \ldots, m-1 \text { for } \beta .\end{cases}
$$

Figures 3 and 4 depict the graphs of cubic and quartic GT-Bézier curves, respectively. Points marked on the cubic GT-Bézier curves relate to $S(0.2)$ in red, $S(0.4)$ in blue, $S(0.6)$ in black, and $S(0.8)$ in green, as well as on quartic GTBézier curves correlate to $S(0.2)$ in orange, $S(0.4)$ in blue, $S(0.6)$ in red, and $S(0.8)$ in green. From these figures, it can be concluded that, by just altering one shape parameter, the points on these curves change in a linear manner.

4.2. Affiliation among the Shape Parameters and the Shape of the Curves. The above defined characteristics of the GTbasis functions make the shape of GT-Bézier curves extraordinarily easier to modify. The appearance of the GTBézier curves can be attuned by changing the values of shape parameters. Figure 5 displays the graphs of cubic, quartic, and quintic GT-Bézier curves. Figure 5(a) shows the cubic GT-Bézier curves with $(\alpha, \beta)=\{(-0.5,-1) \quad$ (purple), $(0.5,-0.5)$ (blue dotted), $(1,0)$ (blue), $(1,0.5)$ (red dashed), $(1,1)$ (black) . The second flower presented in Figure $5(\mathrm{~b})$ is designed by cubic GT-Bézier curves when $(\alpha, \beta)=\{(0,0)$ (red), (1, 1) (green), (-1,-1) (blue), $(0.5,0.5)$ (black dashed), (-0.5,-0.5) (red dotted)\}. Figure 5(c) shows the flower created by quartic GT-Bézier curves with shape parameters $(\alpha, \beta)=\{(-1,-1)$ (red dashed), $(-1,-0.5)$ (blue), $(1,1)$ (black dotted), $(-1,0.5)$ (blue dashed), $(-1,1)$ (orange) . By using different values of shape parameters, $(\alpha, \beta)=\{(0,0)$ (black dotted), (1, 1) (red), $(-1,-1)$ (blue dashed), $(0.5,0.5)$ (orange), $(-0.5,-0.5)$ (purple) $\}$, the quintic GT-Bézier flowers are generated in Figure 5(d). Figure 6(a) represents the shape modification of an apple by changing the values of shape parameters as for $(\alpha, \beta)=\{(1,1)$ (purple), $(0.5,0.5)$ (blue dotted), (0.0) (red dashed dashed), $(-0.5,-0.5)$ (black) , whereas Figure 6(b) illustrates different shapes of the butterfly by applying these values of shape parameters $(\alpha, \beta)=$ $\{(0.9,0.9)$ (black), $(0.5,0.5)$ (orange), $(0.1,0.1)$ (blue) $\}$.

\section{Continuity of GT-Bézier Curves}

The continuity conditions for connecting two GT-Bézier curve segments are described as follows:

Lemma 1 (see [16]). For two given GT-Bézier curves $S(z)=$ $\sum_{i=0}^{m} P_{i} w_{i, m}(z)$ and $S_{1}(z)=\sum_{j=0}^{n} P 1_{j} w_{j, n}(z)$ with control points $P_{0}, P_{1}, P_{2}, \ldots, P_{m}, m \geq 3$, and $P 1_{0}, P 1_{1}, P 1_{2}, \ldots, P 1_{n}$, $n \geq 3$, respectively, the necessary and sufficient constraints for parametric continuity are given by

(1) $P_{m}=P 1_{0}$ for $C^{0}$ continuity

(2) $P_{m}=P 1_{0}$ and $S^{\prime}(1)=S_{1}^{\prime}(0)$ for $C^{1}$ continuity

(3) $P_{m}=P 1_{0}, S^{\prime}(1)=S_{1}^{\prime}(0)$, and $S^{\prime \prime}(1)=S_{1}^{\prime \prime}(0)$ for $C^{2}$ continuity

(4) $P_{m}=P 1_{0}, S^{\prime}(1)=S_{1}^{\prime}(0), S^{\prime \prime}(1)=S_{1}^{\prime \prime}(0)$, and $S^{\prime \prime \prime}(1)$ $=S_{1}^{\prime \prime}(0)$ for $C^{3}$ continuity

Lemma 2 (see [16]). Given control points $P_{0}, P_{1}, P_{2}, \ldots, P_{m}$, $m \geq 3$, and $P 1_{0}, P 1_{1}, P 1_{2}, \ldots, P 1_{n}, n \geq 3$, the necessary and sufficient constraints for connecting two GT-Bézier curve segments $S(z)=\sum_{i=0}^{m} P_{i} w_{i, m}(z)$ and $S_{1}(z)=\sum_{j=0}^{n} P 1_{j} w_{j, n}(z)$ are defined by

(1) For $G^{0}$ continuity: $P_{m}=P 1_{0}$.

(2) For $G^{1}$ continuity: $P_{m}=P 1_{0}$ and $S^{\prime}(1)=\gamma S_{1}^{\prime}(0)$, $\gamma>0$.

(3) For $G^{2}$ continuity: $P_{m}=P 1_{0}, S^{\prime}(1)=\gamma S_{1}^{\prime}(0), \gamma>0$, and the curvature

$$
\kappa(1)=\frac{\left|S^{\prime}(1) \times S^{\prime \prime}(1)\right|}{\left|S^{\prime}(1)\right|^{3}}=\frac{\left|S_{1}^{\prime}(0) \times S_{1}^{\prime \prime}(0)\right|}{\left|S_{1}^{\prime}(0)\right|^{3}}=\kappa_{1}(0) .
$$

Parametric and geometric continuity constraints for connecting two GT-Bézier curve segments are given in the following two theorems.

Theorem 1. For two GT-Bézier curve segments $S(z ; \alpha, \beta)=$ $\sum_{i=0}^{m} P_{i} w_{i, m}(z)$ and $S_{1}(z ; \alpha 1, \beta 1)=\sum_{j=0}^{n} P 1_{j} w_{j, n}(z)$ with control points $P_{0}, P_{1}, P_{2}, \ldots, P_{m}, m \geq 3$, and $P 1_{0}, P 1_{1}$, 


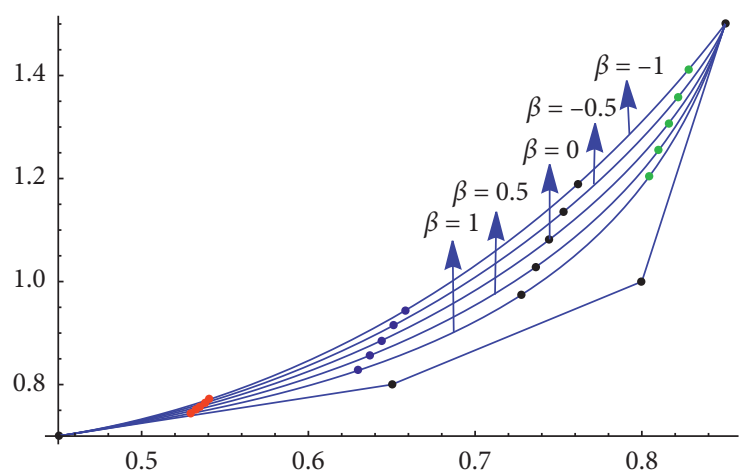

(a)

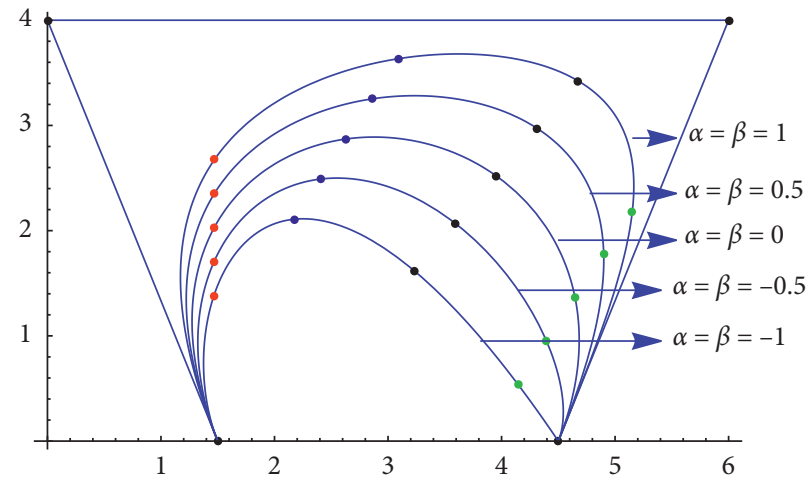

(c)

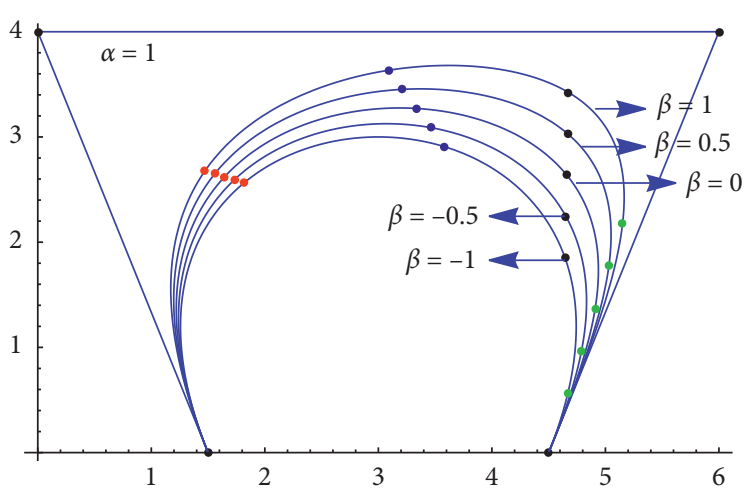

(b)

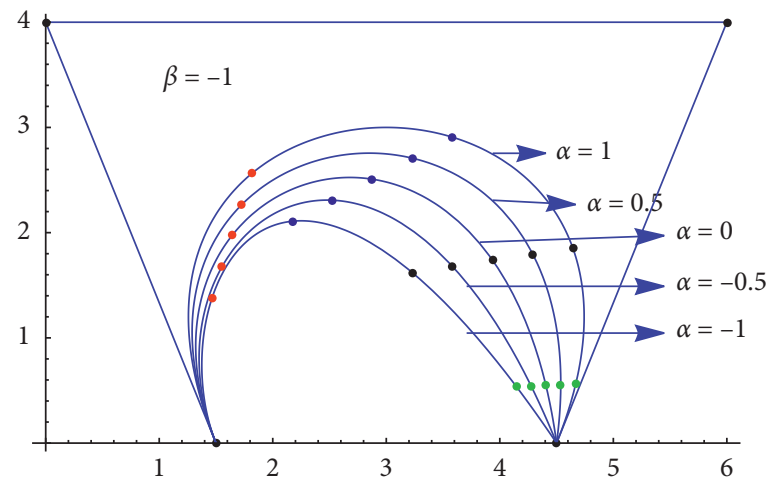

(d)

FIgURE 3: Modifying sequel of shape parameters on the cubic GT-Bézier curve. (a) $\alpha=-1$. (b) $\alpha=1$. (c) $\alpha=\beta$. (d) $\beta=-1$.

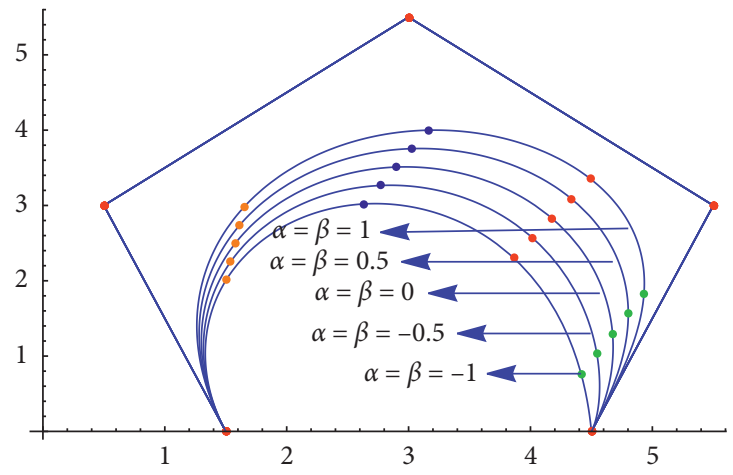

(a)

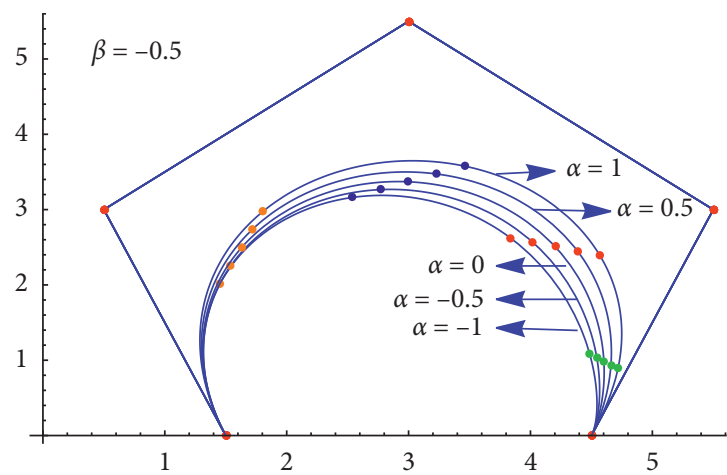

(c)

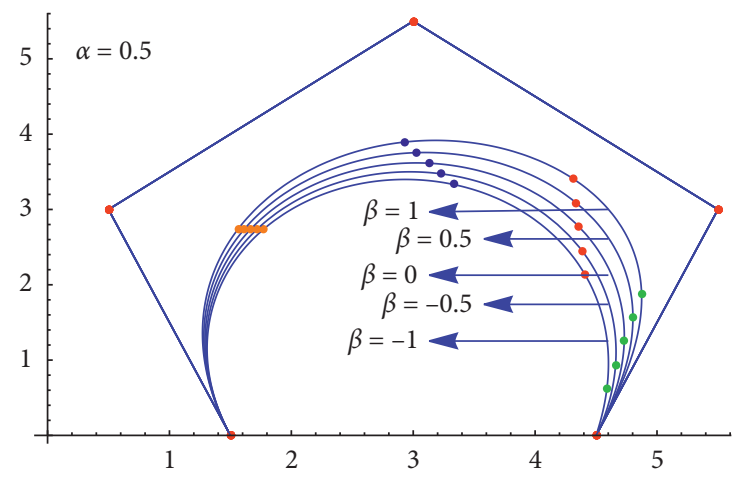

(b)

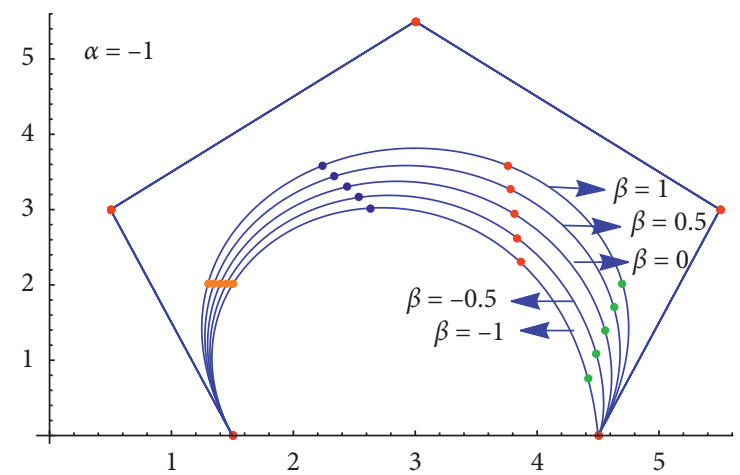

(d)

Figure 4: Effect of shape parameters on quartic GT-Bézier curves. (a) $\alpha=\beta$. (b) $\alpha=0.5$. (c) $\beta=-0.5$. (d) $\alpha=-1$. 


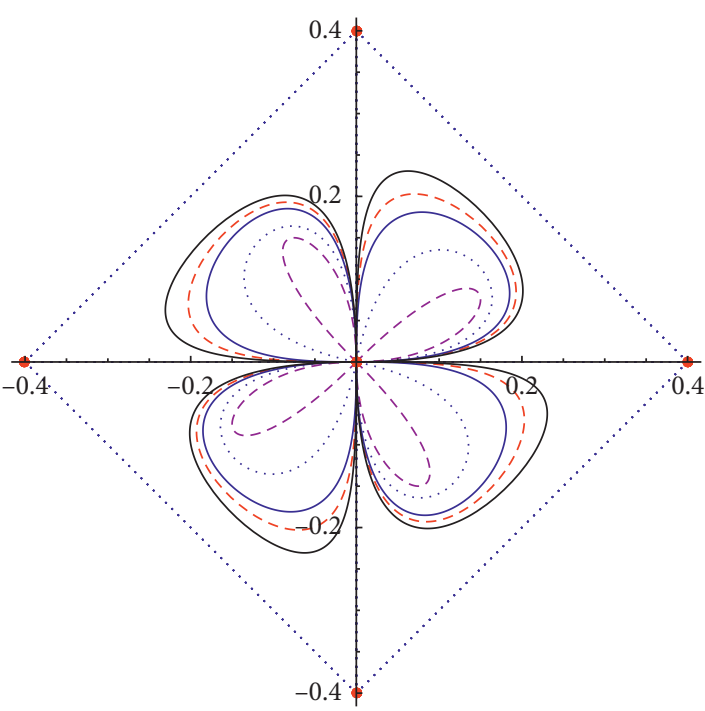

(a)

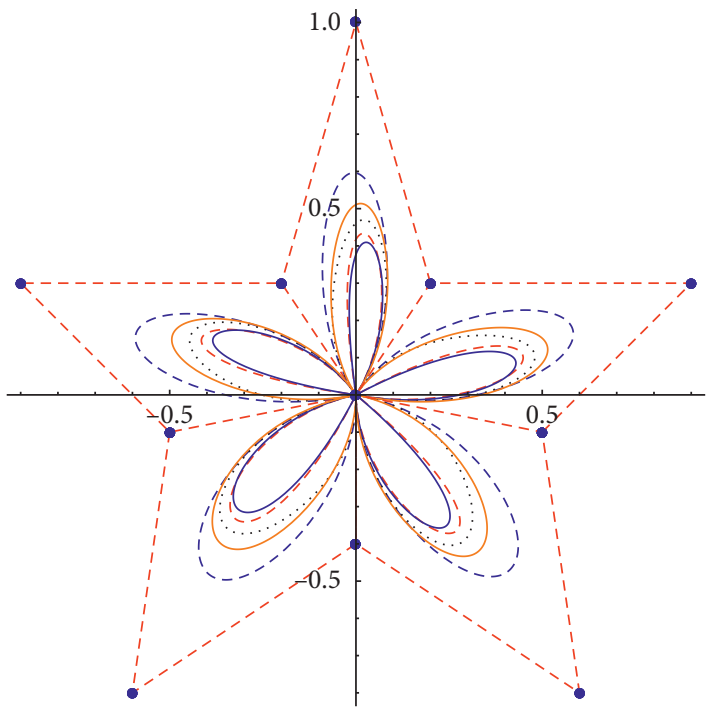

(c)

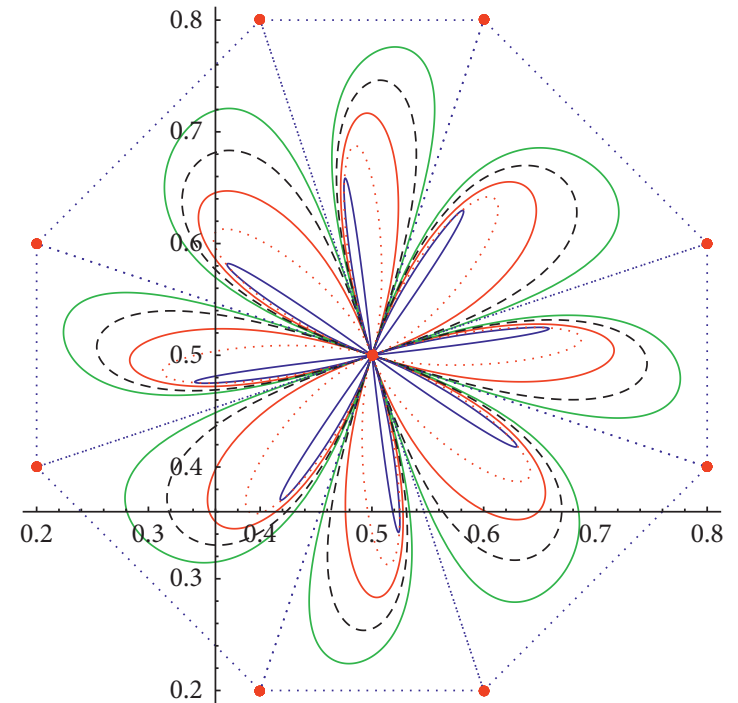

(b)

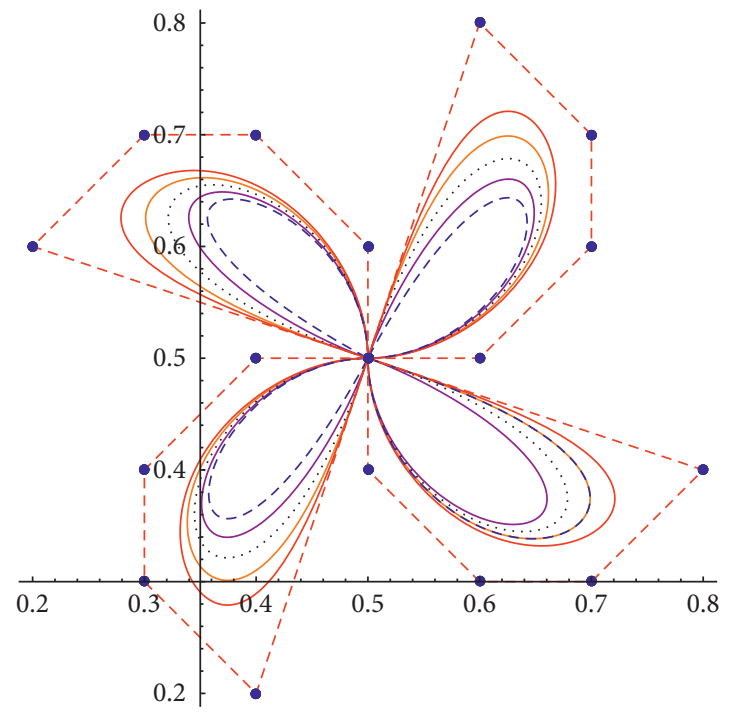

(d)

Figure 5: Flower design by GT-Bézier curves with different shape parameters. (a) $m=3$. (b) $m=3$. (c) $m=4$. (d) $m=5$.

$P 1_{2}, \ldots, P 1_{n}, n \geq 3$, respectively, the necessary and sufficient constraints of parametric continuity are derived as

(1) $C^{0}$ continuity, $P 1_{0}=P_{m}$.

(2) For $C^{1}$ continuity,

$$
\left\{\begin{array}{l}
P 1_{0}=P_{m}, \\
P 1_{1}=P_{m}+\frac{(1+\beta)\left(P_{m}-P_{m-1}\right)}{n_{1}+\alpha 1} .
\end{array}\right.
$$

(3) For $C^{2}$ continuity,

$$
\left\{\begin{array}{l}
P 1_{0}=P_{m}, \\
P 1_{1}=P_{m}+\frac{(1+\beta)\left(P_{m}-P_{m-1}\right)}{n_{1}+\alpha 1}, \\
P 1_{2}=P_{m}+\frac{1}{n_{1} n_{2}+2 n_{2} \alpha 1+(1-\beta 1)}\left[(\alpha-1)\left(P_{m-1}-P_{m-2}\right)\right. \\
\left.-\left(\left(m_{2}-2 \beta\right)-a\left(2 n_{1} n_{2}+\left(2 n_{2}-1\right) 2 \alpha 1+(1-\beta 1)\right)\right)\left(P_{m}-P_{m-1}\right)\right] .
\end{array}\right.
$$

(4) For $C^{3}$ continuity, 


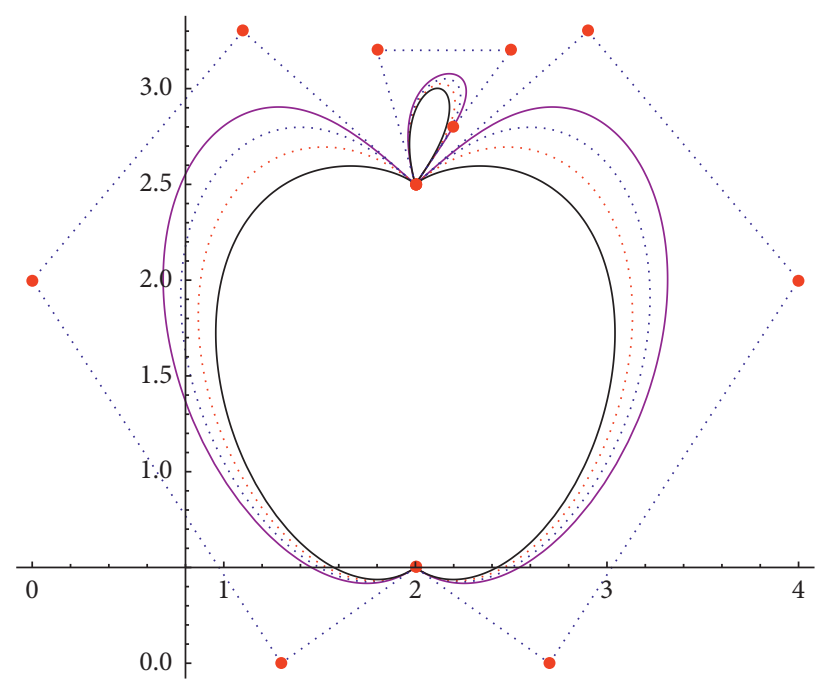

(a)

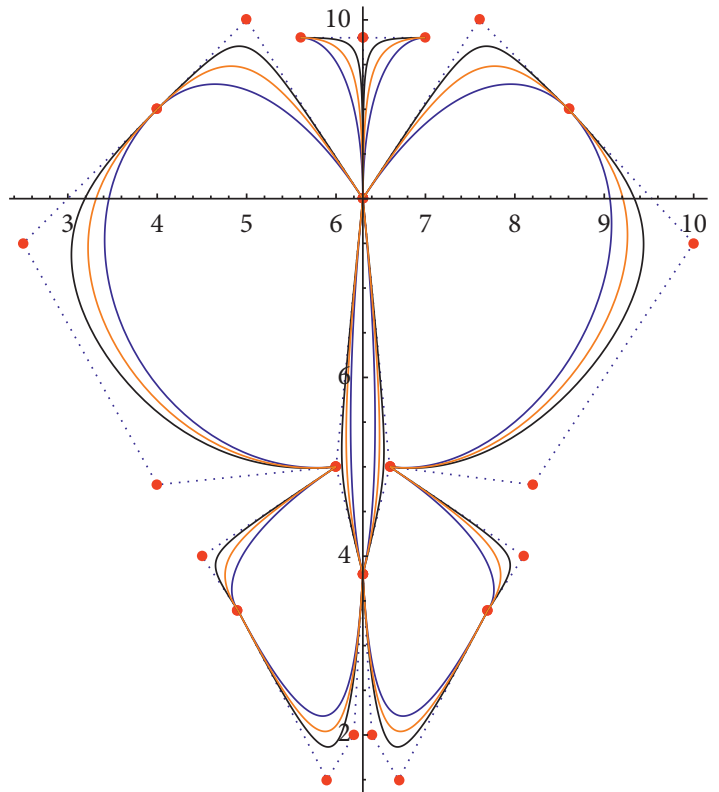

(b)

FIGURE 6: GT-Bézier curve design with different shape parameters.

$$
\left\{\begin{array}{l}
P 1_{0}=P_{m} \\
P 1_{1}=P_{m}+\frac{(1+\beta)\left(P_{m}-P_{m-1}\right)}{n_{1}+\alpha 1}, \\
P 1_{2}=P_{m}+\frac{1}{n_{1} n_{2}+2 n_{2} \alpha 1+(1-\beta 1)}\left[(\alpha-1)\left(P_{m-1}-P_{m-2}\right)-\left(\left(m_{2}-2 \beta\right)-a\left(2 n_{1} n_{2}+\left(2 n_{2}-1\right) 2 \alpha 1+(1-\beta 1)\right)\right)\left(P_{m}-P_{m-1}\right)\right], \\
P 1_{3}=P_{m}+\frac{1}{a_{1}}\left[(1+\beta)\left(P_{m-1}-P_{m-2}\right)-3 m_{2}(1+\beta)\left(P_{m}-2 P_{m-1}+P_{m-2}\right)+a\left(a_{2}-2 n_{1} n_{2} n_{3}-3 n_{2}(1-\beta 1)+6 n_{2}^{2} \alpha 1\right)\left(P_{m}-P_{m-1}\right)\right. \\
\left.\quad+\left(6 n_{2}(1-\beta 1)+3 n_{1} n_{2} n_{3}+n_{2}\left(3 n_{2}-1\right) \alpha 1\right) a_{3}\right],
\end{array}\right.
$$

where $a=\left((1+\beta) /\left(n_{1}+\alpha 1\right)\right), \quad a_{1}=n_{2}\left(3\left(1-\beta 1+n_{1} n_{3}+\right.\right.$ $\left.\left.3 n_{3} \alpha 1\right)\right), a_{2}=\left(n_{3}^{3}-2 n_{2}-1+\left(3 n_{1} n_{2}-1\right) \alpha\right), a_{3}=\left(1 /\left(n_{1} n_{2}+\right.\right.$ $\left.\left.2 n_{2} \alpha 1+(1-\beta 1)\right)\right)\left[(\alpha-1)\left(P_{m-1}-P_{m-2}\right)-\left(\left(m_{2}-2 \beta\right)\right)(-a\right.$ $\left.\left(2 n_{1} n_{2}+\left(2 n_{2}-1\right) 2 \alpha 1+(1-\beta 1)\right)\left(P_{m}-P_{m-1}\right)\right]$, and $n_{k}=$ $(n-k)(k=1,2,3)$.

Proof

(1) $C^{0}$ continuity is simple and straightforward from $S(1)=S_{1}(0)$.

(2) From $C^{0}$ continuity condition, $P_{m}=P 1_{0}$ and $S^{\prime}(1)=S_{1}^{\prime}(0)$. It is simple to obtain the required value of control $P 1_{1}$ from $S^{\prime}(1)=(\pi / 2)(1+\beta)\left(P_{m}-\right.$ $\left.P_{m-1}\right), S_{1}^{\prime}(0)=(\pi / 2)\left(n_{1}+\alpha 1\right)\left(P 1_{1}-P 1_{0}\right)$.

(3) $C^{2}$ continuity condition is $C^{1}$ continuity condition in addition with $S^{\prime \prime}(1)=S_{1}^{\prime \prime}(0)$, so from $P_{m}=P 1_{0}$,
$P 1_{1}=P_{m}+\left((1+\beta)\left(P_{m}-P_{m-1}\right) /\left(n_{1}+\alpha 1\right)\right), \quad$ and end constraints (10), $C^{2}$ continuity constraint (16) is achieved.

(4) $C^{3}$ continuity can be achieved by using $C^{2}$ continuity conditions along with $S^{\prime \prime \prime}(1)=S^{\prime \prime \prime}(0)$. From the terminal properties (9)-(11) of the GT-Bézier curves, $C^{3}$ continuity condition (17) is obtained.

Theorem 2. For two segments of GT-Bézier curves $S(z ; \alpha, \beta)=\sum_{i=0}^{m} P_{i} w_{i, m}(z) \quad$ and $\quad S_{1}(z ; \alpha 1, \beta 1)=\sum_{j=0}^{n}$ $P 1_{j} w_{j, n}(z)$ with control points $P_{0}, P_{1}, P_{2}, \ldots, P_{m}, m \geq 3$, and $P 1_{0}, P 1_{1}, P 1_{2}, \ldots, P 1_{n}, n \geq 3$, the necessary and sufficient constraints of geometric continuity are defined as

(1) $G^{0}$ continuity, $P 1_{0}=P_{m}$.

(2) For $G^{1}$ continuity, 


$$
\left\{\begin{array}{l}
P 1_{0}=P_{m} \\
P 1_{1}=P_{m}+\frac{(1+\beta)\left(P_{m}-P_{m-1}\right)}{\left(n_{1}+\alpha 1\right) \gamma} .
\end{array}\right.
$$

(3) For $G^{2}$ continuity,

$$
\left\{\begin{array}{l}
P 1_{0}=P_{m} \\
P 1_{1}=P_{m}+\frac{(1+\beta)\left(P_{m}-P_{m-1}\right)}{\left(n_{1}+\alpha 1\right) \gamma}, \\
P 1_{2}=P_{m}+\frac{1}{\gamma^{2}\left(n_{1} n_{2}+2 n_{2} \alpha 1+(1-\beta 1)\right.} \\
\left.\quad\left[(\alpha-1)\left(P_{m-1}-P_{m-2}\right)-\left(\left(m_{2}-2 \beta\right)-b\left(2 n_{1} n_{2}+\left(2 n_{2}-1\right) 2 \alpha 1+(1-\beta 1)-\frac{2 \lambda}{\pi}\left(n_{1}+\alpha 1\right)\right)\right)\right)\left(P_{m}-P_{m-1}\right)\right]
\end{array}\right.
$$

where $b=\left((1+\beta) / \gamma\left(n_{1}+\alpha 1\right)\right)$.

Proof

(1) Using straightforward computations, $G^{0}$ continuity is obvious.

(2) $G^{1}$ continuity condition can be achieved if $P 1_{0}=P_{m}$ and $S^{\prime}(1)=\gamma S_{1}^{\prime}(0), \gamma>0$. Using end constraints $S^{\prime}(1)=(\pi / 2)(1+\beta)\left(P_{m}-P_{m-1}\right) \quad$ and, $S_{1}^{\prime}(0)=(\pi / 2)\left(n_{1}+\alpha 1\right)\left(P 1_{1}-P 1_{0}\right)$. From the terminal properties of the GT-Bézier curves, $G^{1}$ continuity condition (18) is obtained.

(3) If both GT-Bézier curves $S(z ; \alpha, \beta)$ and $S_{1}(z ; \alpha 1, \beta 1)$ connect by $G^{2}$ continuity, they need to connect first by $G^{1}$ at a common joint, which means

$$
\left\{\begin{array}{l}
S(1 ; \alpha, \beta)=P_{m}=Q_{0}=S_{1}(0 ; \alpha 1, \beta 1), \\
S^{\prime}(1)=\gamma S_{1}^{\prime}(0), \quad \gamma>0 .
\end{array}\right.
$$

Suppose that the vice normal vector $N_{1}=S^{\prime}(1) \times S^{\prime \prime}(1)$ for $S(z)$ at $z=1$ and reverse normal vector $N_{2}=S_{1}^{\prime}(0) \times$ $S_{1}^{\prime \prime}(0)$ of $S_{1}(z)$; then, we have

$$
\left\{\begin{array}{l}
N_{1}=S^{\prime}(1) \times S^{\prime \prime}(1), \\
N_{2}=S_{1}^{\prime}(0) \times S_{1}^{\prime \prime}(0) .
\end{array}\right.
$$

Both vice normal vectors $N_{1}$ and $N_{2}$ must have the same direction at the joint to achieve the $G^{2}$ continuity. Four vectors $S^{\prime}(1), S^{\prime \prime}(1), S_{1}^{\prime}(0)$, and $S_{1}^{\prime \prime}(0)$ become coplanar by combining equations (20) and (21). Thus, we obtain

$$
S^{\prime \prime}(1)=\delta S_{1}^{\prime \prime}(0)+\lambda S_{1}^{\prime}(0), \quad \delta>0 .
$$

As $G^{2}$ continuity is archived, if the curvatures $\kappa_{1}(t)$ and $\kappa_{2}(t)$ for the GT-Bézier curves $S(z ; \alpha, \beta)$ and $S_{1}(z ; \alpha 1, \beta 1)$, respectively, have the same value at the joint point, i.e., $\kappa_{1}(1)=\kappa_{2}(0)$, using equations (20)-(22), we obtain

$$
\begin{aligned}
\kappa_{1}(1) & =\frac{\left|S^{\prime}(1) \times S^{\prime \prime}(1)\right|}{\left|S^{\prime}(1)\right|^{3}} \\
& =\frac{\left|\gamma S_{1}^{\prime}(0) \times\left(\delta S_{1}^{\prime \prime}(0)+\lambda S_{1}^{\prime}(0)\right)\right|}{\gamma^{3}\left|S_{1}^{\prime}(0)\right|^{3}} \\
& =\frac{\delta\left|S_{1}^{\prime}(0) \times S_{1}^{\prime \prime}(0)\right|}{\gamma^{2}\left|S_{1}^{\prime}(0)\right|^{3}} \\
& =\kappa_{2}(0) .
\end{aligned}
$$

From equation (23), $\delta=\gamma^{2}$, and substituting this value into equation (22), we have

$$
S^{\prime \prime}(1)=\gamma^{2} S_{1}^{\prime \prime}(0)+\lambda S_{1}^{\prime}(0) .
$$

From the terminal properties of the GT-Bézier curves, $G^{2}$ continuity condition (19) is obtained.

\section{Examples and Discussions}

Some examples and discussions about parametric and geometric continuity of GT-Bézier curves are given in this section.

(1) Figure 7(a) illustrates the $C^{1}$ continuity of GT-Bézier curves of order 3, 4, and 5, respectively, with control points $P_{0}=(0.1,0.4), P_{1}=(0.15,0.75), P_{2}=(0.25$, $0.9), \quad P_{3}=(0.5,0.9), \quad P_{4}=(0.5,0.6)=P 1_{0}, \quad P 1_{1}=$ $(0.5,0.4), P 1_{2}=(0.65,0.3), P 1_{3}=(0.8,0.35), P 1_{4}=$ $(0.9,0.5), P 1_{5}=(0.85,0.7)=P 2_{0}, P 2_{1}=(0.8,0.9)$, $P 2_{2}=(0.95,1.05)$, and $P 2_{3}=(1.1,0.8)$ for different values of shape parameters $\alpha, \beta$. Applying $C^{1}$ continuity conditions (15) on these GT-Bézier curves, points $P 1_{1}$ and $P 2_{1}$ for every curve can be derived as $(0.5,0.48),(0.5,0.5),(0.5,0.25)$, and $(0.5,0.557)$ and $(0.817,0.83),(0.82,0.82),(0.825,0.8)$, and 


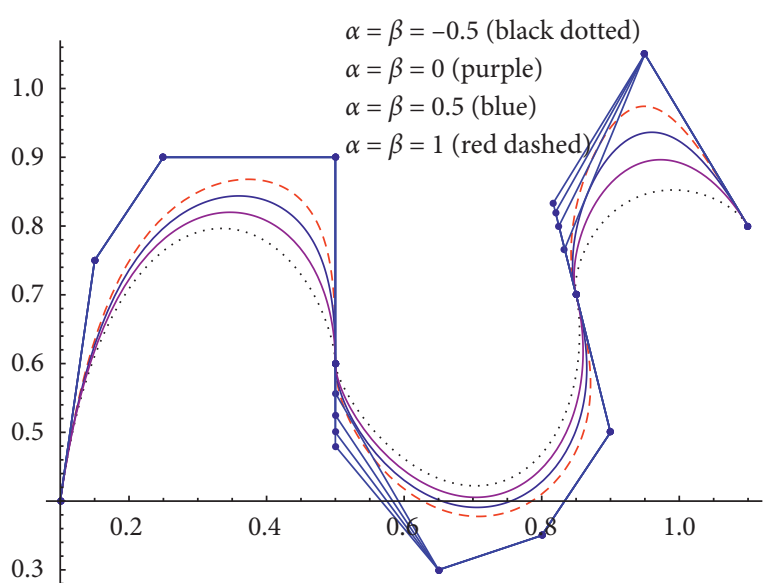

(a)

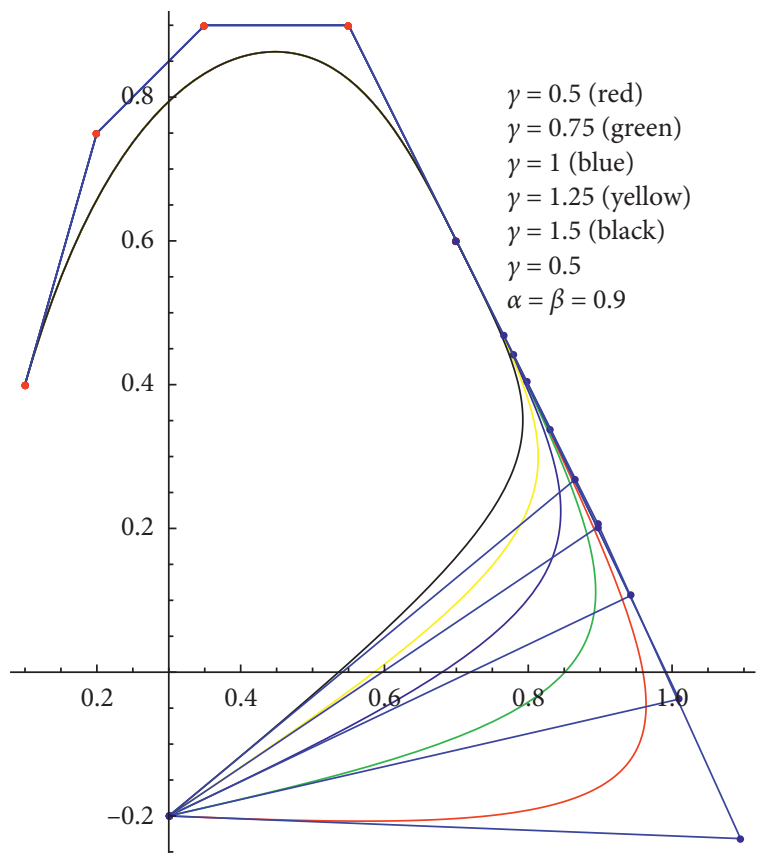

(c)

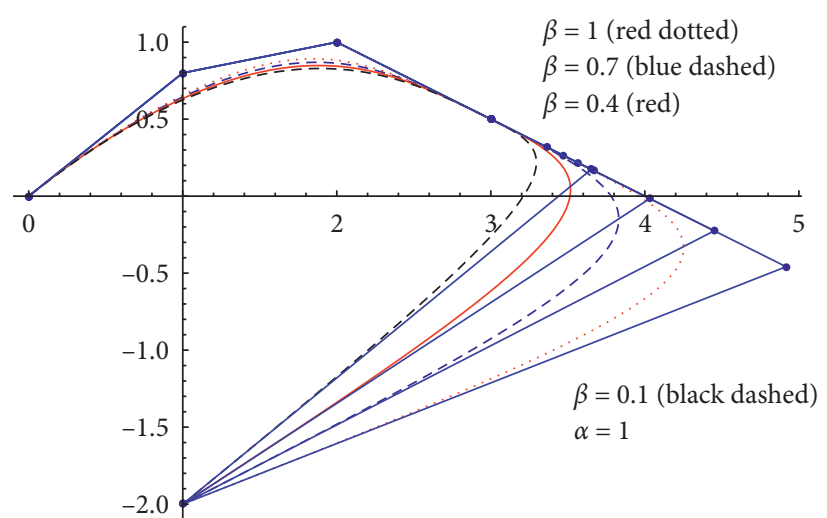

(b)

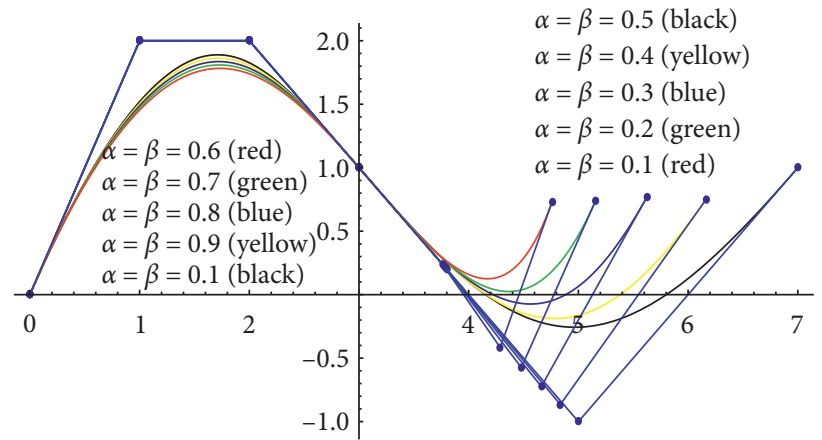

(d)

Figure 7: Parametric and geometric GT-Bézier continuity curves of different degrees. (a) $C^{1}$ continuity. (b) $C^{2}$ continuity. (c) $G^{2}$ continuity. (d) $C^{3}$ continuity.

$(0.833,0.766)$, respectively, with decreasing values of $\alpha, \beta$.

(2) For given control points $P_{0}=(0,0), P_{1}=(1,0.8)$, $P_{2}=(2,1), P_{3}=(3,0.5)=P 1_{0}$, and $P 1_{3}=(1,-2)$, Figure $7(\mathrm{~b})$ represents $C^{2}$ continuity of two cubic GT-Bézier curve segments. Using $C^{2}$ continuity constraints (16) of two GT-Bézier curves, the calculated control points $P 1_{1}$ and $P 1_{2}$ for each curve according to the decreasing values of $\beta$ are $(3.66,0.16),(3.6,0.2),(3.45,0.27)$, and $(3.36,0.3)$ and $(4.9,-0.46),(4.5,-0.22),(4,-0.015)$, and $(3.6,0.17)$, respectively.

(3) Figure 7(c) displays the $G^{2}$ continuity of cubic and quartic GT-Bézier curves with control points $P_{0}=(0.1,0.4), \quad P_{1}=(0.2,0.75), \quad P_{2}=(0.35,0.9)$,
$P_{3}=(0.55,0.9), \quad P_{4}=(0.7,0.6)=P 1_{0}, \quad$ and $P 1_{3}=(0.3,-0.2)$ and shape parameters $\alpha, \beta$. Using conditions (19) of $G^{2}$ continuity from Theorem 2 , the computed control points $P 1_{1}$ and $P 1_{2}$ for each curve corresponding to the increasing value of $\gamma$ are given as $(0.89,0.2),(0.83,0.33),(0.79,0.4),(0.78,0.44)$, and $(0.76,0.46)$ and $(1.09,-0.23),(1,-0.03)$, $(0.94,0.1),(0.89,0.2)$, and $(0.86,0.26)$, respectively.

(4) Two cubic GT-Bézier curves exhibit $C^{3}$ continuity in Figure $7(\mathrm{~d})$ along with control points $P_{0}=(0,0)$, $P_{1}=(1,2), P_{2}=(2,2)$, and $P_{3}=(3,1)=P 1_{0}$ and shape parameters $\alpha, \beta$. Using conditions (17) of $C^{3}$ continuity from Theorem 1, calculated control point $P 1_{1}$ for each curve is $(3.8,0.2),(3.79,0.21)$, $(3.78,0.22),(3.77,0.23)$, and $(3.76,0.24)$, point $P 1_{2}$ is $(5,-1),(4.8,-0.86),(4.6,-0.7),(4.8,-0.5)$, and 
$(4.3,-0.41)$, and point $P 1_{3}$ is $(7,1),(6.1,0.75)$, $(5.6,0.77), \quad(5,0.73), \quad$ and $(4.7,0.733)$ correspondingly.

\section{GT-Bézier Surfaces}

7.1. Formation of the GT-Bézier Surface. The GT-Bézier surfaces of order $m \times n$ for given points $P_{i, j} \in R^{2}$ or $R^{3}(i=$ $0,1, \ldots, m, j=0,1, \ldots, n) \quad$ with shape parameters $\alpha, \alpha 1, \beta, \beta 1$ are defined as

$$
W_{m, n}\left(z, z_{1}\right)=\sum_{i=0}^{m} \sum_{j=0}^{n} P_{i, j} w_{i, m}(z) w_{j, n}\left(z_{1}\right), \quad z, z_{1} \in[0,1],
$$

where $w_{i, m}(z), w_{j, n}\left(z_{1}\right)(i=0,1, \ldots, m, j=0,1, \ldots, n)$ are GT-basis functions (2). Figures $8(\mathrm{a})-8(\mathrm{~d})$ represent the GTBézier vine glass, GT-Bézier vase diagrams, and GT-Bézier swept surface of different orders but with fixed values of shape parameters, whereas Figures 9(a)-9(c) demonstrate the GT-Bézier surfaces of different degrees with different values of shape parameters.

7.2. $G^{2}$ Continuity of GT-Bézier Surfaces. In engineering applications, $G^{2}$ continuity between two adjacent surface patches is required, that is, at every point where the curved surfaces meet, the surfaces must have a combined tangent plane or a collective normal line to the surface $[3-5,38]$. There exists continuity in different four types of directions between two GT-Bézier surfaces $W_{m 1, n 1}\left(z, z_{1} ; \alpha, \alpha 1, \beta, \beta 1\right)=$ $\sum_{i=0}^{m 1} \sum_{j=0}^{n 1} P_{i, j} w_{i, m 1}(z) w_{j, n 1}\left(z_{1}\right) \quad$ and $W 1_{m 2, n 2}\left(z, z_{1} ; \alpha^{*}\right.$, $\left.\alpha 1^{*}, \beta^{*}, \beta 1^{*}\right)=\sum_{k=0}^{m 2} \sum_{l=0}^{n 2} P 1_{k, l} w_{k, m 2}(z) w_{l, n 2}\left(z_{1}\right)$.
7.3. Continuity in $z$ Direction on Every Surface. Two regions of GT-Bézier surfaces should have a collective edge curve to attain $G^{1}$ continuity, i.e.,

$$
W_{m 1, n 1}(z, 1 ; \alpha, \alpha 1, \beta, \beta 1)=W 1_{m 2, n 2}\left(z, 0 ; \alpha^{*}, \alpha 1^{*}, \beta^{*}, \beta 1^{*}\right),
$$

which results in

$$
\sum_{i=0}^{m_{1}} P_{i, n_{1}} w_{i, m_{1}}(z ; \alpha, \beta)=\sum_{k=0}^{m_{2}} P 1_{k, 0} w_{k, m_{2}}\left(z ; \alpha^{*}, \beta^{*}\right),
$$

implying

$$
\sum_{i=0}^{m} P_{i, n_{1}} w_{i, m}(z ; \alpha, \beta)=\sum_{i=0}^{m} P 1_{i, 0} w_{i, m}\left(t ; \alpha^{*}, \beta^{*}\right) .
$$

If $\alpha=\alpha^{*}, \beta=\beta^{*}$, then

$$
P_{i, n_{1}}=P 1_{i, 0} \text {. }
$$

The normal direction at the boundary is uninterrupted for two regions of the surfaces, i.e., the joining boundary should have a mutual tangent plane to meet the $G^{1}$ continuity between two regions of the surfaces. So, we have the following condition:

$$
\begin{aligned}
& \frac{\partial}{\partial z} W_{m, n_{1}}(z, 1 ; \alpha, \alpha 1, \beta, \beta 1) \times \frac{\partial}{\partial z_{1}} W_{m, n_{1}}(z, 1 ; \alpha, \alpha 1, \beta, \beta 1) \\
& \quad=f\left(z_{1}\right) \frac{\partial}{\partial z} W 1_{m, n_{2}}\left(z, 0 ; \alpha^{*}, \alpha 1^{*}, \beta^{*}, \beta 1^{*}\right) \times \frac{\partial}{\partial z_{1}},
\end{aligned}
$$$$
W 1_{m, n_{2}}\left(t, 0 ; \alpha^{*}, \alpha 1^{*}, \beta^{*}, \beta 1^{*}\right) \text {, }
$$

where $f\left(z_{1}\right)$ is the scaling factor among their normal vectors such that $f\left(z_{1}\right)>0$. Equivalently,

$$
\frac{\partial}{\partial z_{1}} W_{m, n_{1}}(z, 1 ; \alpha, \alpha 1, \beta, \beta 1)=f \cdot \frac{\partial}{\partial z_{1}} W 1_{m, n_{2}}\left(z, 0 ; \alpha^{*}, \alpha 1^{*}, \beta^{*}, \beta 1^{*}\right) .
$$

Since

$$
\begin{aligned}
\frac{\partial}{\partial z_{1}} W_{m, n_{1}}(z, 1 ; \alpha, \alpha 1, \beta, \beta 1) & =\frac{\pi}{2} \sum_{i=0}^{m} w_{i, m}(z ; \alpha, \beta)(1+\beta 1)\left(P_{i, n_{1}}-P_{i, n_{1}-1}\right), \\
\frac{\partial}{\partial z_{1}} W 1_{m, n_{2}}\left(z, 0 ; \alpha^{*}, \alpha 1^{*}, \beta^{*}, \beta 1^{*}\right) & =\frac{\pi}{2} \sum_{i=0}^{m} w_{i, m}\left(z ; \alpha^{*}, \beta^{*}\right)\left(n_{2}-1+\alpha 1^{*}\right)\left(P 1_{i, 1}-P 1_{i, 0}\right) .
\end{aligned}
$$

Substituting (32) and (33) into equation (31), we have

$$
\begin{aligned}
& \frac{\pi}{2} \sum_{i=0}^{m} w_{i, m}(z ; \alpha, \beta)(1+\beta 1)\left(P_{i, n_{1}}-P_{i, n_{1}-1}\right) \\
& \quad=f \cdot \frac{\pi}{2} \sum_{i=0}^{m} w_{i, m}\left(z ; \alpha^{*}, \beta^{*}\right)\left(n_{2}-1+\alpha 1^{*}\right)\left(P 1_{i, 1}-P 1_{i, 0}\right) .
\end{aligned}
$$

$$
\begin{aligned}
& \text { If } \alpha=\alpha^{*}, \beta=\beta^{*} \text {, then } \\
& (1+\beta 1)\left(P_{i, n_{1}}-P_{i, n_{1}-1}\right)=f \cdot\left(n_{2}-1+\alpha 1^{*}\right)\left(P 1_{i, 1}-P 1_{i, 0}\right) .
\end{aligned}
$$

Continuity conditions on each surface are described in equations (29) and (35) which are the general conditions for $G^{1}$ continuity in $z$ direction. In accumulation, under $G^{1}$ continuity condition, the two surfaces need to retain the similar normal curvature at any point on their joint 


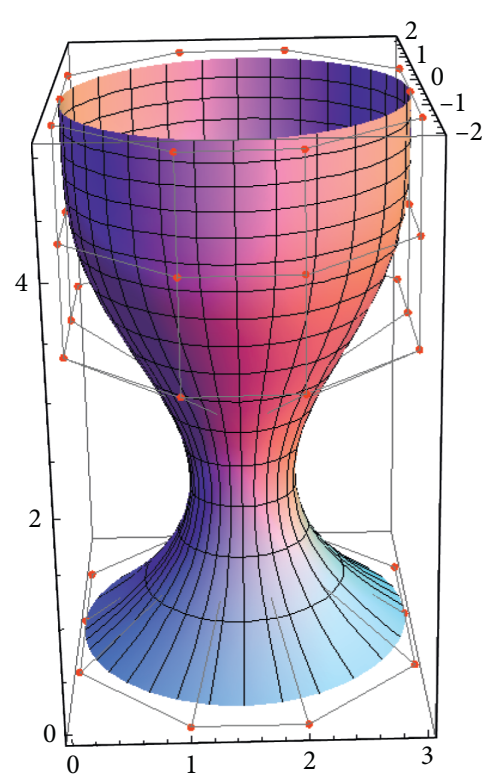

(a)

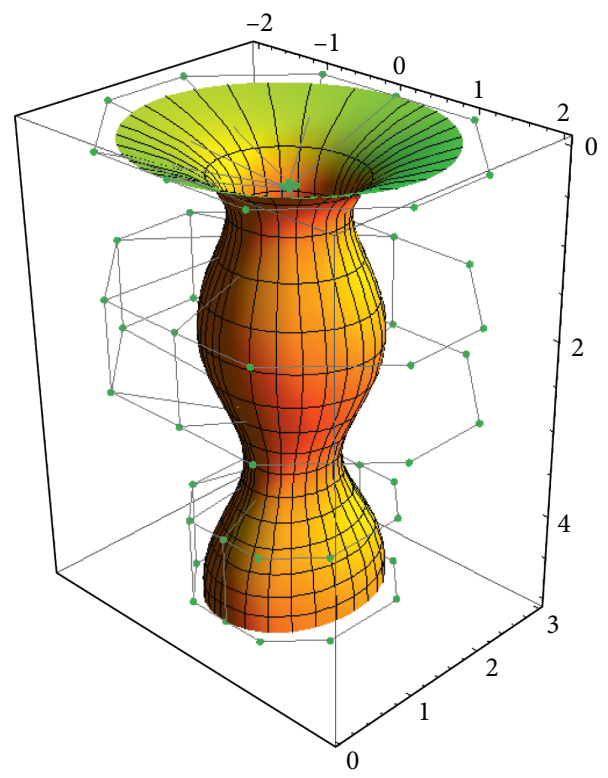

(c)

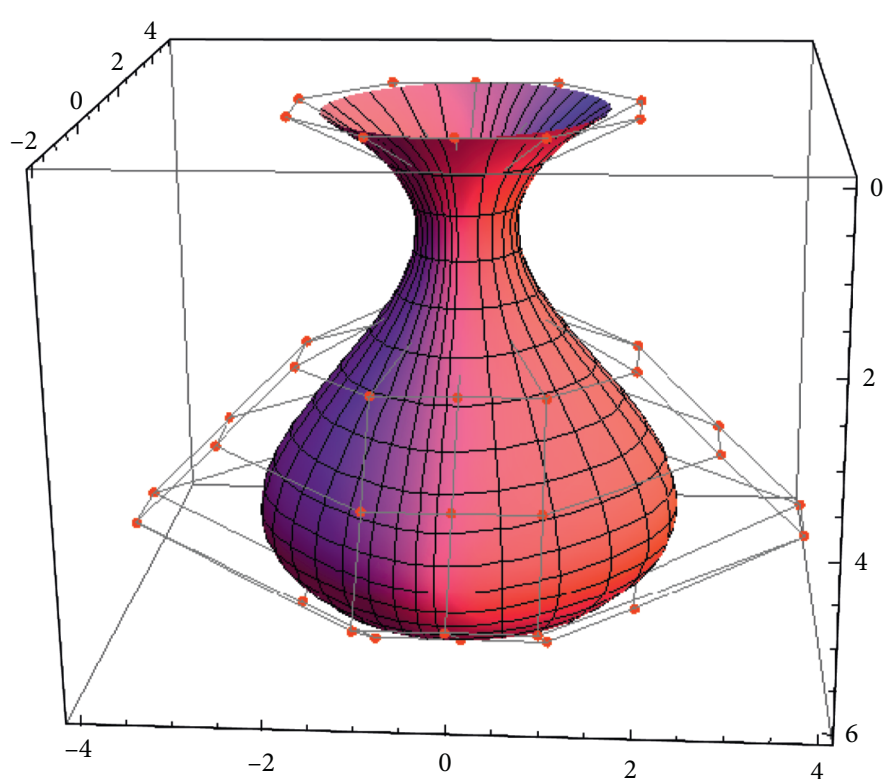

(b)

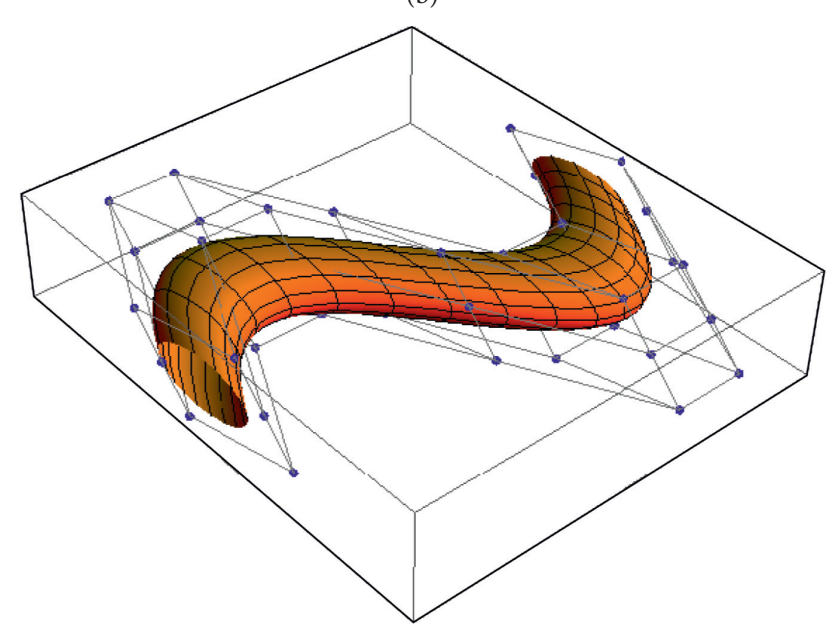

(d)

FIGURE 8: GT-Bézier surfaces of different degrees. (a) 5th order GT-Bézier surface. (b) $(5 \times 6)$ th order GT-Bézier surface. (c) $(8 \times 5)$ th order GT-Bézier surface. (d) 6th order GT-Bézier surface. 
boundary, so the two surfaces also have to fulfil the following $G^{2}$ continuity constraints:

$$
\begin{aligned}
\frac{\partial^{2}}{\partial z_{1}^{2}} W_{m, n_{1}}(z, 1 ; \alpha, \alpha 1, \beta, \beta 1)= & f^{2} \frac{\partial^{2}}{\partial z_{1}^{2}} W 1_{m, n_{2}}\left(z, 0 ; \alpha^{*}, \alpha 1^{*}, \beta^{*}, \beta 1^{*}\right)+2 f g(z) \frac{\partial}{\partial z \partial z_{1}} W 1_{m, n_{2}}\left(z, 0 ; \alpha^{*}, \alpha 1^{*}, \beta^{*}, \beta 1^{*}\right) \\
& +g^{2}(z) \frac{\partial^{2}}{\partial z_{1} \partial z_{1}} W 1_{m, n_{2}}\left(z, 0 ; \alpha^{*}, \alpha 1^{*}, \beta^{*}, \beta 1^{*}\right)+c \frac{\partial}{\partial z_{1}} W 1_{m, n_{2}}\left(z, 0 ; \alpha^{*}, \alpha 1^{*}, \beta^{*}, \beta 1^{*}\right) \\
& +d(z) \frac{\partial}{\partial z} W 1_{m, n_{2}}\left(z, 0 ; \alpha^{*}, \alpha 1^{*}, \beta^{*}, \beta 1^{*}\right)
\end{aligned}
$$

where $g(z)$ and $d(z)$ are linear functions of $z$ and $c$ is a random constant. For the simplification of operation and calculation in applied submissions, generally, set $g(z)=d(z)=c=0$ [32], so the above equation can be further simplified as

$$
\begin{aligned}
& \frac{\partial^{2}}{\partial z_{1}^{2}} W_{m, n_{1}}(z, 1 ; \alpha, \alpha 1, \beta, \beta 1) \\
& \quad=f^{2} \frac{\partial^{2}}{\partial z_{1}^{2}} W 1_{m, n_{2}}\left(z, 0 ; \alpha^{*}, \alpha 1^{*}, \beta^{*}, \beta 1^{*}\right) .
\end{aligned}
$$

Since

$$
\begin{gathered}
\frac{\partial^{2}}{\partial z_{1}^{2}} W_{m, n_{1}}(z, 1 ; \alpha, \alpha 1, \beta, \beta 1)=\frac{\pi^{2}}{4} \sum_{i=0}^{m} w_{i, m}(z ; \alpha, \beta)\left[(1-\alpha 1)\left(P_{i, n_{1}-2}-P_{i, n_{1}-1}\right)\right. \\
\left.+\left(n_{1}-2(1+\beta 1)\right)\left(P_{i, n_{1}-1}-P_{i, n_{1}}\right)\right], \\
\frac{\partial^{2}}{\partial z_{1}^{2}} W 1_{m, n_{2}}\left(z, 0 ; \alpha^{*}, \alpha 1^{*}, \beta^{*}, \beta 1^{*}\right)=\frac{\pi^{2}}{4} \sum_{i=0}^{m} w_{i, m}\left(z ; \alpha^{*}, \beta^{*}\right)\left[\left(\left(n_{2}-1\right)\left(n_{2}-2\right)+2 \alpha 1^{*}\right)\left(P 1_{i, 0}-P 1_{i, 1}\right)\right. \\
\left.-\left(\left(n_{2}-1\right)\left(n_{2}-2\right)+2\left(n_{2}-2\right) \alpha 1^{*}+\left(1-\beta 1^{*}\right)\right)\left(P 1_{i, 1}-P 1_{i, 2}\right)\right] .
\end{gathered}
$$

Substituting (38) and (39) into equation (37), we get

$$
\begin{aligned}
& \frac{\pi^{2}}{4} \sum_{i=0}^{m} w_{i, m}(z ; \alpha, \beta)\left[(1-\alpha 1)\left(P_{i, n_{1}-2}-P_{i, n_{1}-1}\right)+\left(n_{1}-2(1+\beta 1)\right)\left(P_{i, n_{1}-1}-P_{i, n_{1}}\right)\right] \\
& =f^{2} \frac{\pi^{2}}{4} \sum_{i=0}^{m} w_{i, m}\left(z ; \alpha^{*}, \beta^{*}\right)\left[\left(\left(n_{2}-1\right)\left(n_{2}-2\right)+2 \alpha 1^{*}\right)\left(P 1_{i, 0}-P 1_{i, 1}\right)-\left(\left(n_{2}-1\right)\left(n_{2}-2\right)\right.\right. \\
& \left.\left.+2\left(n_{2}-2\right) \alpha 1^{*}+\left(1-\beta 1^{*}\right)\right)\left(P 1_{i, 1}-P 1_{i, 2}\right)\right] . \\
& (1-\alpha 1)\left(P_{i, n_{1}-2}-P_{i, n_{1}-1}\right)+\left(n_{1}-2(1+\beta 1)\right)\left(P_{i, n_{1}-1}-P_{i, n_{1}}\right) \\
& =f^{2}\left[\left(\left(n_{2}-1\right)\left(n_{2}-2\right)+2 \alpha 1^{*}\right)\left(P 1_{i, 0}-P 1_{i, 1}\right)-\left(\left(n_{2}-1\right)\left(n_{2}-2\right)\right.\right. \\
& \left.\left.+2\left(n_{2}-2\right) \alpha 1^{*}+\left(1-\beta 1^{*}\right)\right)\left(P 1_{i, 1}-P 1_{i, 2}\right)\right] \text {. }
\end{aligned}
$$

If $\alpha=\alpha^{*}, \beta=\beta^{*}$, we have 

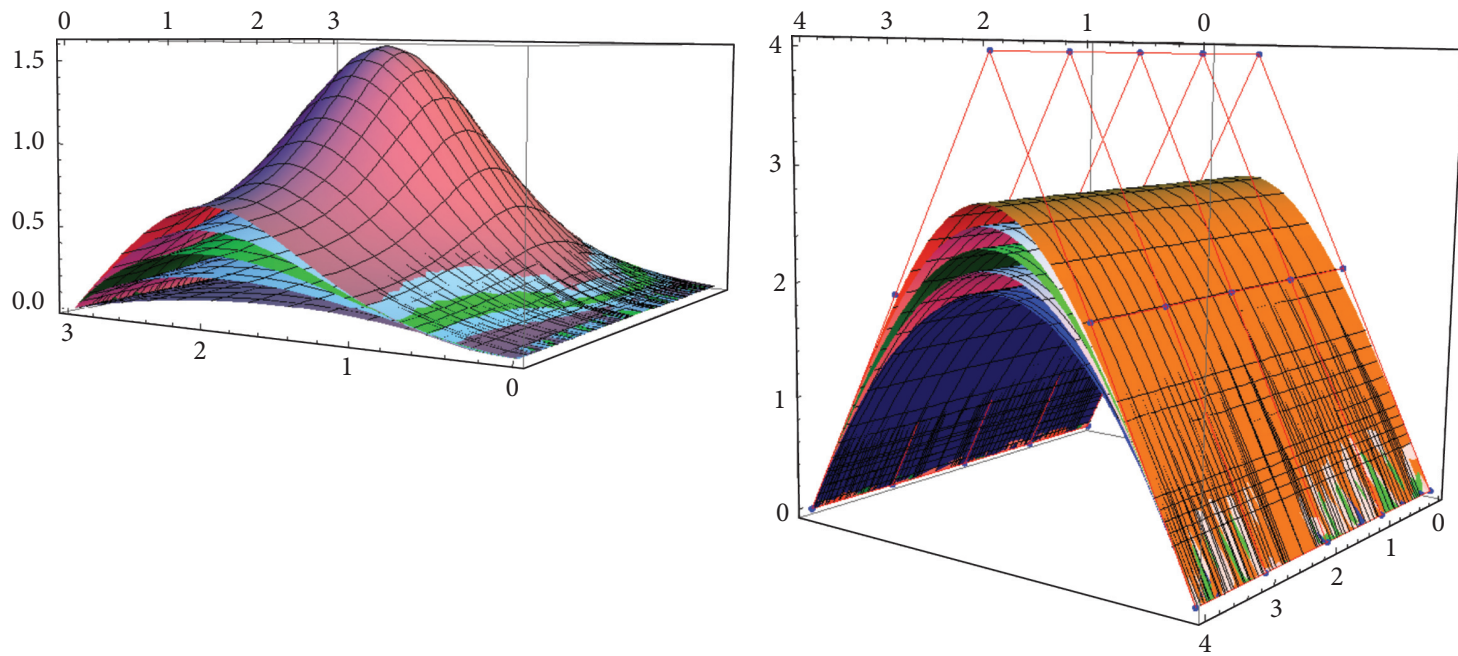

(a)

(b)

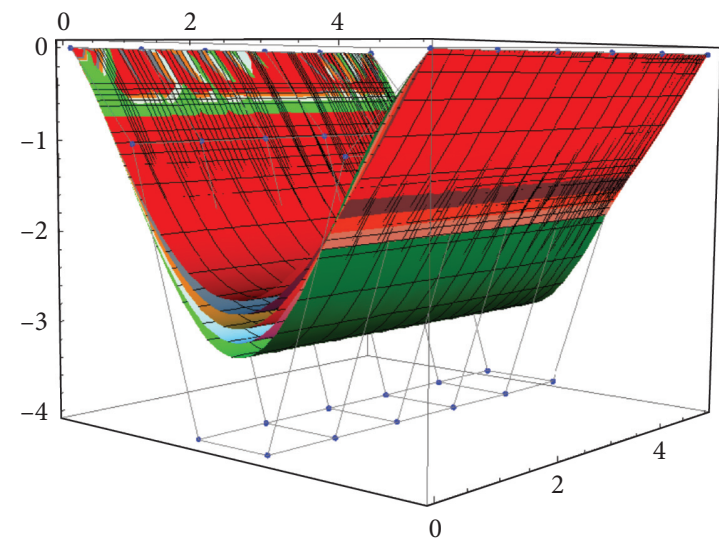

(c)

Figure 9: GT-Bézier surfaces of different degrees with multiple shape parameters. (a) Cubic GT-Bézier surfaces. (b) Quartic GT-Bézier surfaces. (c) Quintic GT-Bézier surfaces.

To sum up, if the two surfaces $W_{m, n_{2}}(z, 1 ; \alpha, \alpha 1, \beta, \beta 1)$ and $W 1_{m, n_{2}}\left(z, 0 ; \alpha^{*}, \alpha 1^{*}, \beta^{*}, \beta 1^{*}\right)$ satisfy (29), (35), and (41) at the same time, the $G^{2}$ continuity in $z$ direction is achieved.

7.4. Continuity in $z$ and $z_{1}$ Direction. The condition of holding a common boundary when the surface $W_{m 1, n 1}\left(z, z_{1} ; \alpha, \alpha 1, \beta, \beta 1\right)$ has continuity in $z$ direction and the surface $W 1_{m 2, n 2}\left(z, z_{1} ; \alpha^{*}, \alpha 1^{*}, \beta^{*}, \beta 1^{*}\right)$ has continuity in $z_{1}$ direction is

$$
W_{m 1, n 1}(z, 1 ; \alpha, \alpha 1, \beta, \beta 1)=W 1_{m 2, n 2}\left(0, z_{1} ; \alpha^{*}, \alpha 1^{*}, \beta^{*}, \beta 1^{*}\right),
$$

or

$$
\sum_{i=0}^{m_{1}} P_{i, n_{1}} w_{i, m_{1}}(z ; \alpha, \beta)=\sum_{l=0}^{n_{2}} P 1_{0, l} w_{l, n_{2}}\left(z_{1} ; \alpha 1^{*}, \beta 1^{*}\right),
$$

implying

$$
\sum_{i=0}^{m} P_{i, n_{1}} w_{i, m}(z ; \alpha, \beta)=\sum_{i=0}^{m} P 1_{0, i} w_{i, m}\left(z_{1} ; \alpha 1^{*}, \beta 1^{*}\right)
$$

If $\alpha=\alpha 1^{*}, \beta=\beta 1^{*}$, then equation (44) is further simplified as

$$
P_{i, n_{1}}=P 1_{0, i}
$$

For having a common tangent plane, we have the following condition:

$$
\begin{aligned}
& \frac{\partial}{\partial z_{1}} W_{m_{1}, n_{1}}(z, 1 ; \alpha, \alpha 1, \beta, \beta 1) \\
& \quad=f \cdot \frac{\partial}{\partial z} W 1_{m_{2}, n_{2}}\left(0, z_{1} ; \alpha^{*}, \alpha 1^{*}, \beta^{*}, \beta 1^{*}\right) .
\end{aligned}
$$

Since 


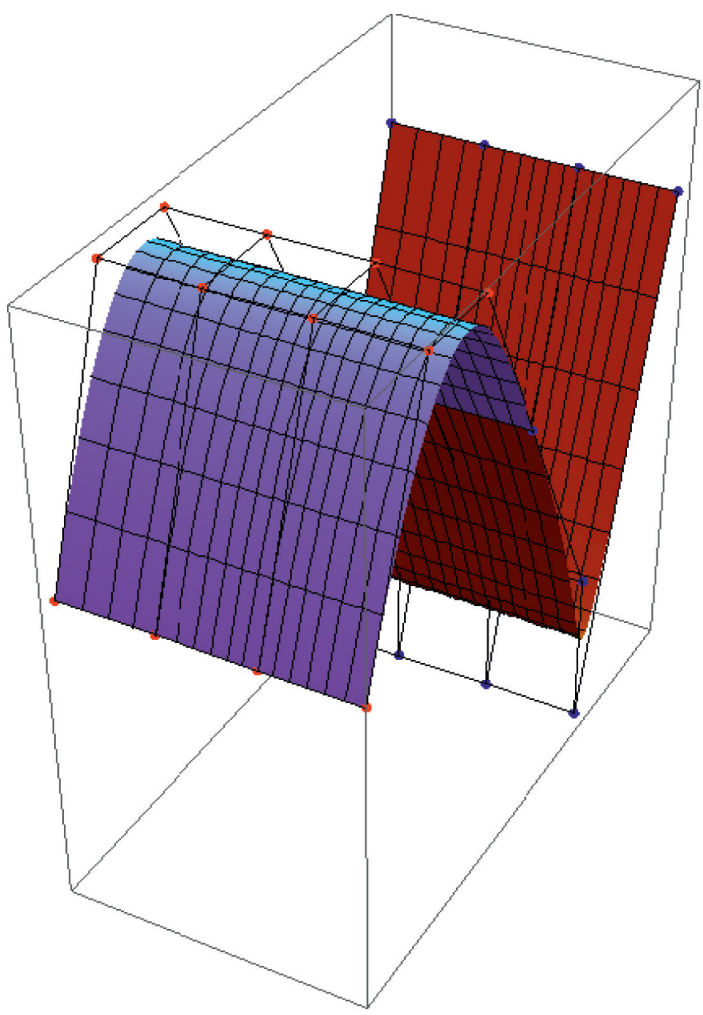

(a)

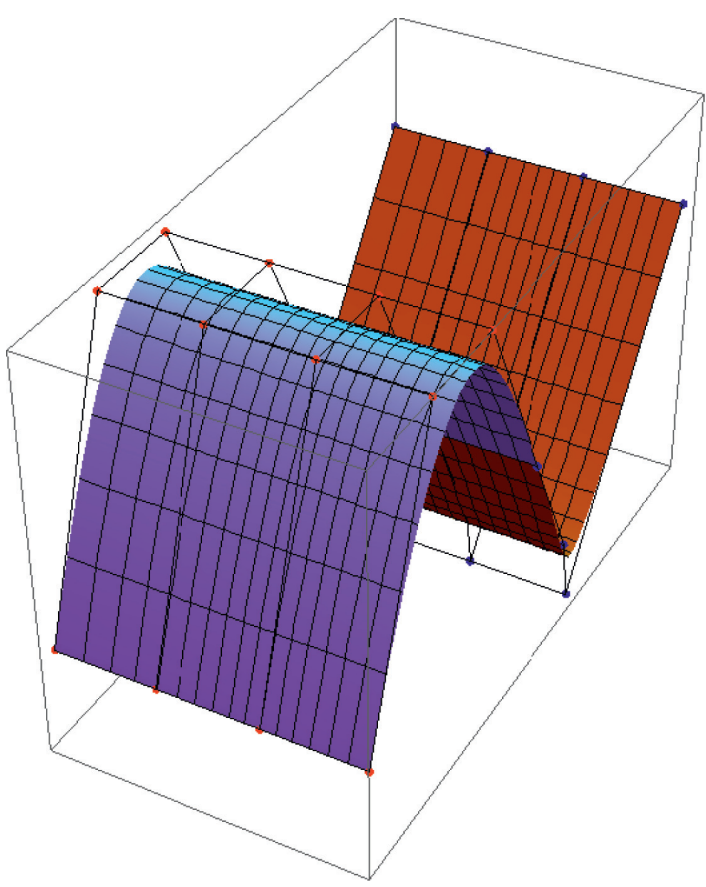

(c)

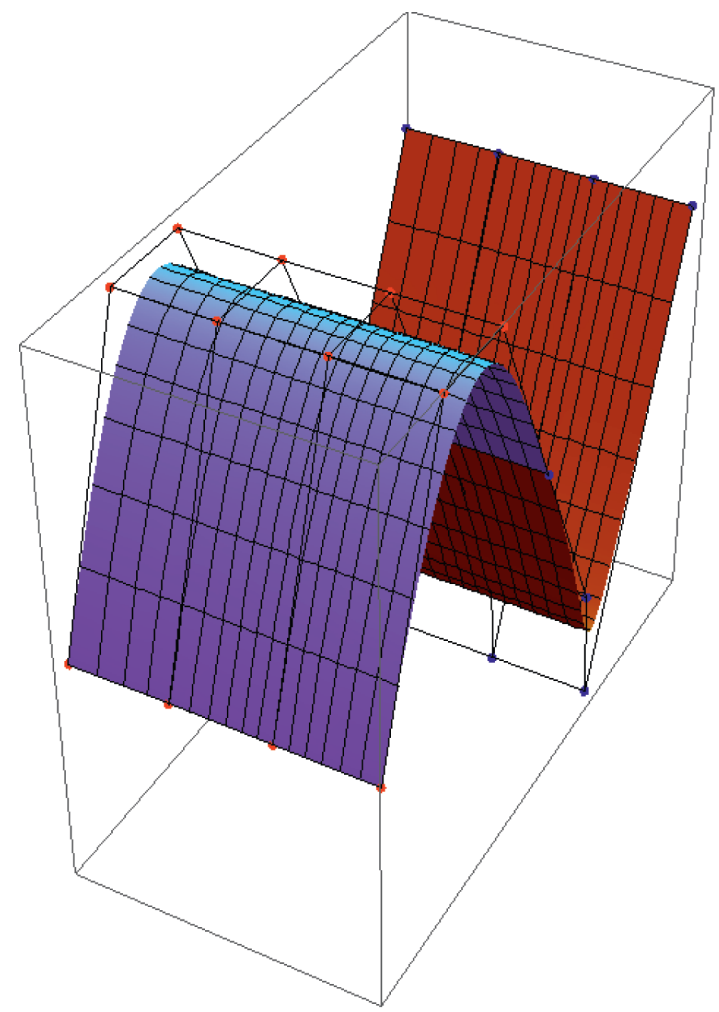

(b)

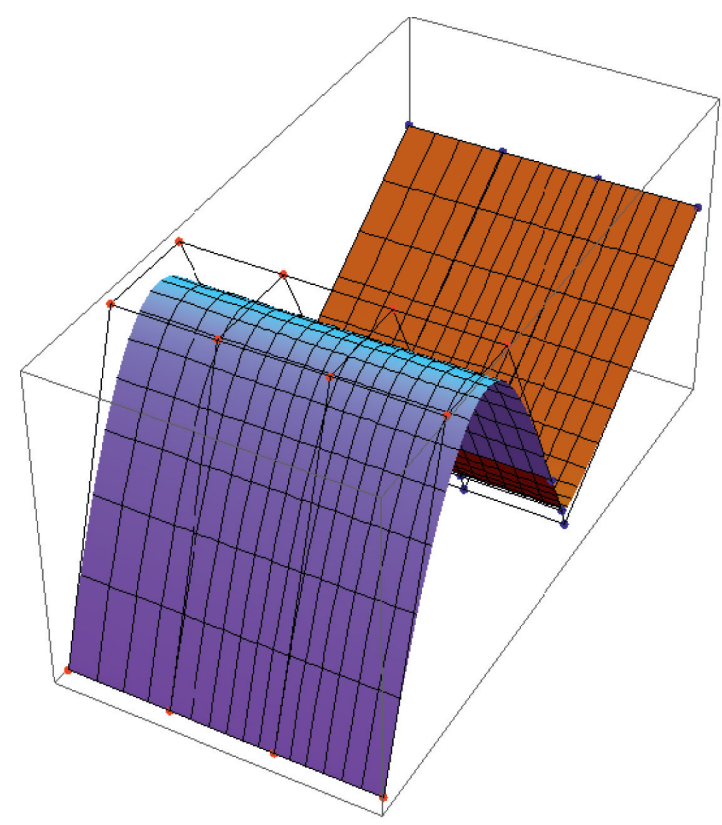

(d)

FIGURE 10: The effect of changing scaling factor on cubic GT-Bézier surfaces. (a) $f=0.75, \alpha, \beta, \alpha^{*}, \beta^{*}=1$. (b) $f=1, \alpha, \beta, \alpha^{*}, \beta^{*}=1$. (c) $f=1.5, \alpha, \beta, \alpha^{*}, \beta^{*}=1$. (d) $f=4, \alpha, \alpha^{*}=1, \beta, \beta^{*}=1$. 


$$
\begin{aligned}
& \frac{\partial}{\partial z_{1}} W_{m_{1}, n_{1}}(z, 1 ; \alpha, \alpha 1, \beta, \beta 1) \\
& \quad=\frac{\pi}{2} \sum_{i=0}^{m_{1}} w_{i, m_{1}}(z ; \alpha, \beta)(1+\beta 1)\left(P_{i, n_{1}}-P_{i, n_{1}-1}\right), \\
& \frac{\partial}{\partial z} W 1_{m_{2}, n_{2}}\left(0, z_{1} ; \alpha^{*}, \alpha 1^{*}, \beta^{*}, \beta 1^{*}\right) \\
& =\frac{\pi}{2} \sum_{l=0}^{n_{2}} w_{l, n_{2}}\left(z_{1} ; \alpha 1^{*}, \beta 1^{*}\right)\left(m_{2}-1+\alpha^{*}\right)\left(P 1_{1, l}-P 1_{0, l}\right) .
\end{aligned}
$$

Substituting (47) and (48) into equation (46), we obtain

$$
\begin{aligned}
& \frac{\pi}{2} \sum_{i=0}^{m_{1}} w_{i, m_{1}}(z ; \alpha, \beta)(1+\beta 1)\left(P_{i, n_{1}}-P_{i, n_{1}-1}\right) \\
& \quad=\frac{\pi}{2} \sum_{l=0}^{n_{2}} P 1_{0, l} w_{l, n_{2}}\left(z_{1} ; \alpha 1^{*}, \beta 1^{*}\right)\left(m_{2}-1+\alpha^{*}\right)\left(P 1_{1, l}-P 1_{0, l}\right) .
\end{aligned}
$$

If $\alpha=\alpha 1^{*}, \beta=\beta 1^{*}$, we have

$$
(1+\beta 1)\left(P_{i, n_{1}}-P_{i, n_{1}-1}\right)=f \cdot\left(m_{2}-1+\alpha^{*}\right)\left(P 1_{1, i}-P 1_{0, i}\right) .
$$

Hence, the general conditions for $G^{1}$ continuity in $z$ and $z_{1}$ direction are described in equations (41) and (46); when $\alpha=\alpha 1^{*}, \beta=\beta 1^{*}$, then the continuity conditions are defined in equations (45) and (50).

Moreover, from $G^{1}$ smooth continuity conditions, the two surfaces are also essential to have the same normal curvature at each point on their shared boundary, so for $G^{2}$ continuity, they also required to satisfy

$$
\begin{aligned}
& \frac{\partial^{2}}{\partial z_{1}^{2}} W_{m, n_{1}}(z, 1 ; \alpha, \alpha 1, \beta, \beta 1) \\
& \quad=f^{2} \frac{\partial^{2}}{\partial z^{2}} W 1_{m, n_{2}}\left(0, z_{1} ; \alpha^{*}, \alpha 1^{*}, \beta^{*}, \beta 1^{*}\right) .
\end{aligned}
$$

Substituting the boundary surfaces into equation (53), we get

$$
\begin{aligned}
& \frac{\pi^{2}}{4} \sum_{i=0}^{m} w_{i, m}(z ; \alpha, \beta)\left[(1-\alpha 1)\left(P_{i, n_{1}-2}-P_{i, n_{1}-1}\right)+\left(n_{1}-2(1+\beta 1)\right)\left(P_{i, n_{1}-1}-P_{i, n_{1}}\right)\right] \\
& \quad=f^{2} \frac{\pi^{2}}{4} \sum_{i=0}^{m} w_{i, m}\left(z_{1} ; \alpha 1^{*}, \beta 1^{*}\right)\left[\left(\left(m_{2}-1\right)\left(m_{2}-2\right)+2 \alpha^{*}\right)\left(P 1_{0, i}-P 1_{1, i}\right)-\left(\left(m_{2}-1\right)\left(m_{2}-2\right)\right.\right. \\
& \left.\left.\quad+2\left(m_{2}-2\right) \alpha^{*}+\left(1-\beta^{*}\right)\right)\left(P 1_{1, i}-P 1_{2, i}\right)\right] .
\end{aligned}
$$

When $\alpha=\alpha 1^{*}, \beta=\beta 1^{*}$, equation (52) yields

$$
\begin{aligned}
& (1-\alpha 1)\left(P_{i, n_{1}-2}-P_{i, n_{1}-1}\right)+\left(n_{1}-2(1+\beta 1)\right)\left(P_{i, n_{1}-1}-P_{i, n_{1}}\right) \\
& \quad=f^{2}\left[\left(\left(m_{2}-1\right)\left(m_{2}-2\right)+2 \alpha^{*}\right)\left(P 1_{0, i}-P 1_{1, i}\right)+\left(\left(m_{2}-1\right)\left(m_{2}-2\right)+2\left(m_{2}-2\right) \alpha^{*}+\left(1-\beta^{*}\right)\right)\left(P 1_{2, i}-P 1_{1, i}\right)\right] .
\end{aligned}
$$

Finally, if the two surfaces $W_{m, n_{2}}(z, 1 ; \alpha, \alpha 1, \beta, \beta 1)$ and $W 1_{m, n_{2}}\left(0, z_{1} ; \alpha^{*}, \alpha 1^{*}, \beta^{*}, \beta 1^{*}\right)$ satisfy (45), (50), and (53), then both surfaces are connected by $G^{2}$ continuity in $z$ and $z_{1}$ direction.

7.5. Continuity in $z_{1}$ Direction. The $G^{1}$ and $G^{2}$ continuity in $z_{1}$ direction of two GT-Bézier surfaces is achieved in a similar fashion as the continuity in $z$ direction of two GTBézier surfaces, which is discussed in Section 7.3.

7.6. Continuity in $z_{1}$ and $z$ Direction. The $G^{1}$ and $G^{2}$ continuity in direction $z_{1}$ and $z$ is obtained in a similar way as the continuity in $z$ and $z_{1}$ direction which is proved in Section 7.4.

The $G^{2}$ continuity between two GT-Bézier surfaces is also analyzed as for two GT-Bézier surfaces, the continuity in $z$ direction is achieved; when $\alpha=\alpha^{*}, \beta=\beta^{*}$, then the $G^{1}$ continuity between two regions of GT-Bézier surfaces has $m_{1}=m_{2}=m$ mutual control points, and the control points $P_{i, n_{1}-1}, P_{i, n_{1}}\left(\right.$ or $\left.P 1_{i, 0}\right), P 1_{i, 1}$ are taken and varied in order. For continuity in $z$ and $z_{1}$ direction, when $\alpha=\alpha 1^{*}, \beta=\beta 1^{*}$, then the $G^{2}$ continuity between two regions of GT-Bézier surfaces has $m_{1}=n_{2}$ mutual control points, and the control points $P_{i, n_{1}-1}, P_{i, n_{1}}$ (or $\left.P 1_{0, i}\right), P 1_{1, i}$ are in the same straight line and changeable in order. 


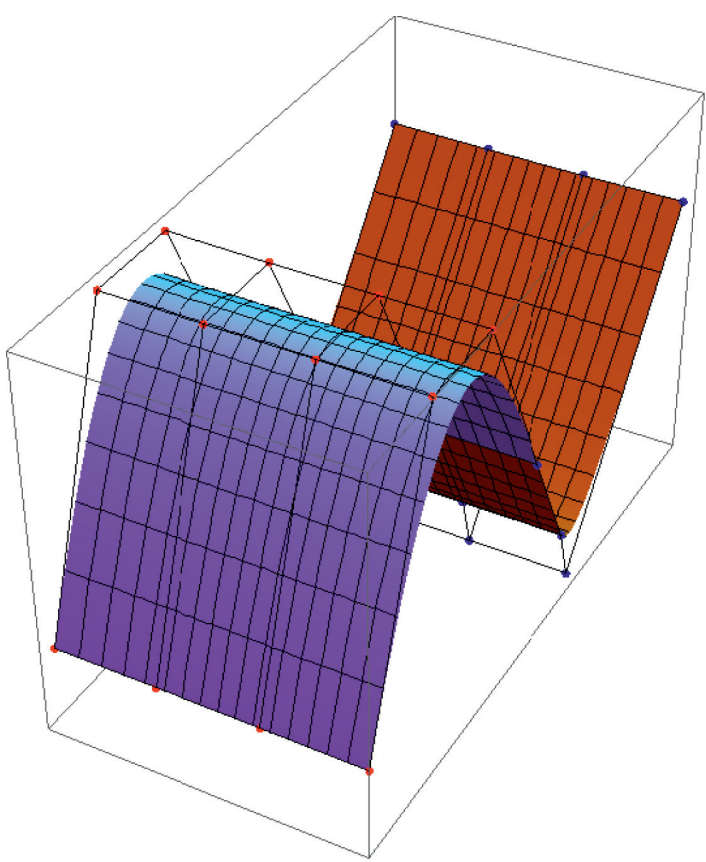

(a)

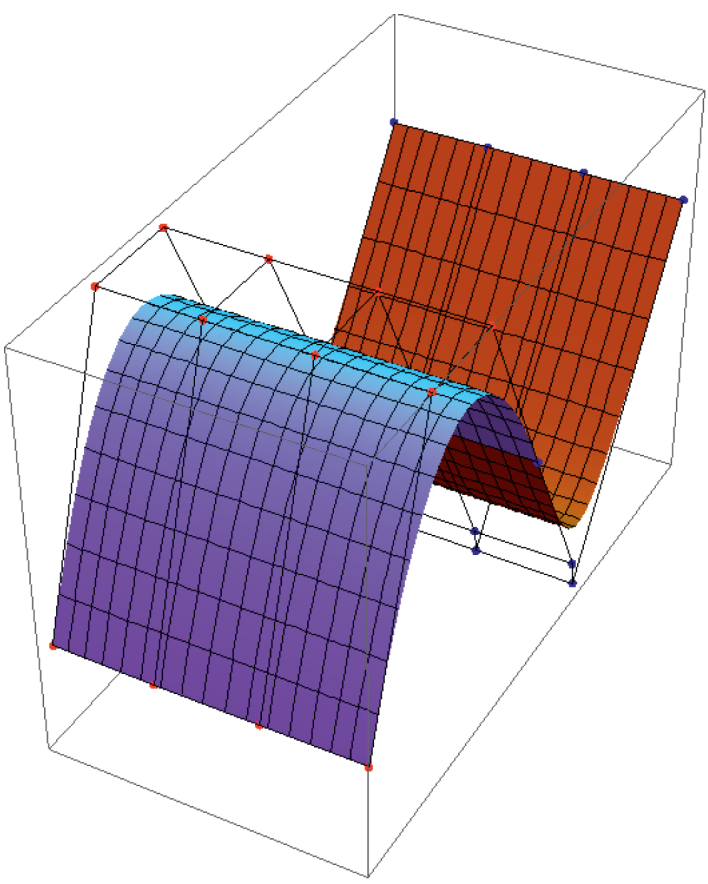

(c)

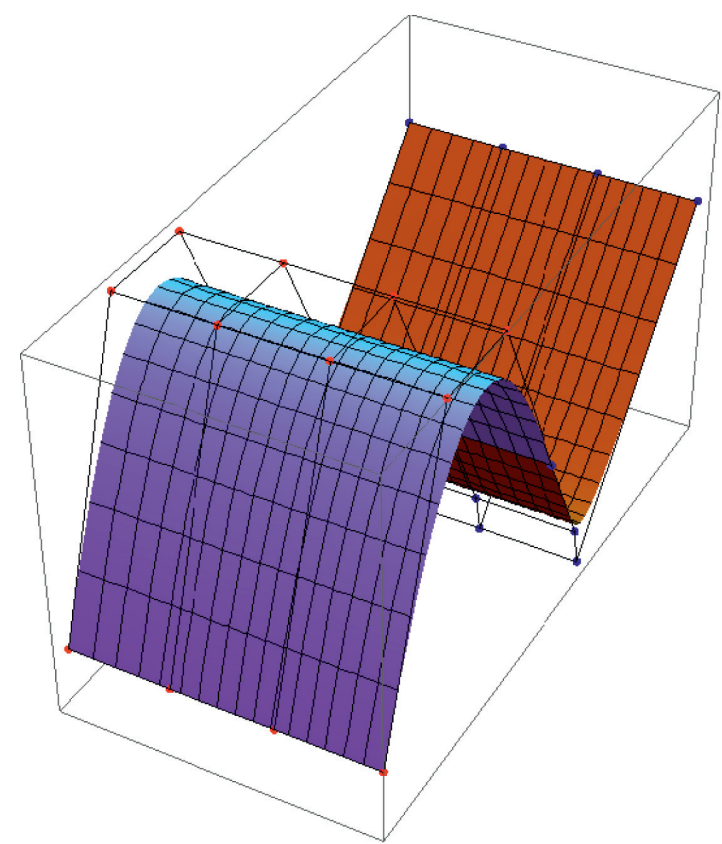

(b)

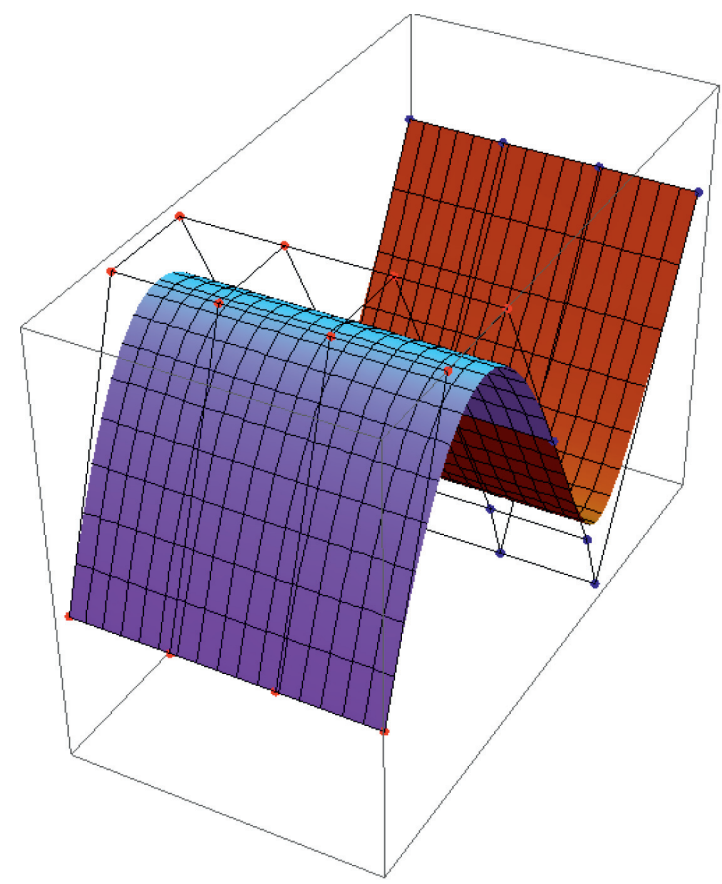

(d)

Figure 11: The effect of shape parameters on cubic GT-Bézier surfaces. (a) $f=2, \alpha, \alpha^{*}=0.5, \beta, \beta^{*}=1$. (b) $f=2, \alpha, \alpha^{*}=0.5, \beta, \beta^{*}=0.85$. (c) $f=2, \alpha, \alpha^{*}=-0.5, \beta, \beta^{*}=0.65$. (d) $f=2, \alpha, \alpha^{*}=-0.35, \beta, \beta^{*}=0.9$.

Figures 10-15 depict the $G^{2}$ continuity surfaces between two GT-Bézier surfaces of different degrees in $z$ direction for different shape parameters and scaling factor $f$. For the fixed values of shape parameters, the control points $P 1_{1, i}$ (or $P 1_{2, i}$ ) move closer to (away from) the control points $P 1_{0, i}\left(\right.$ or $P 1_{1, i}$ ) when thevalues of the scaling factor $f$ increases (or 


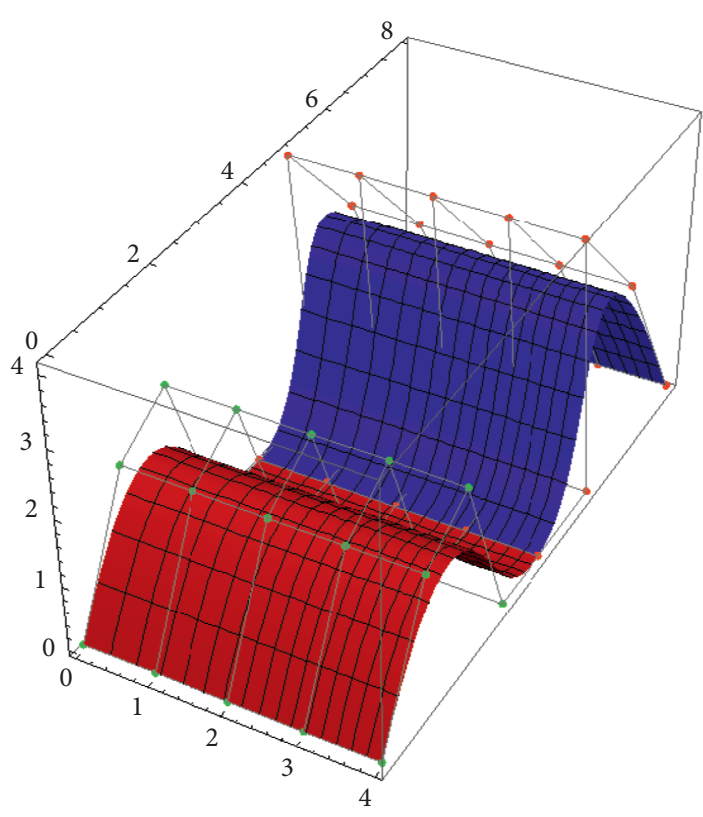

(a)

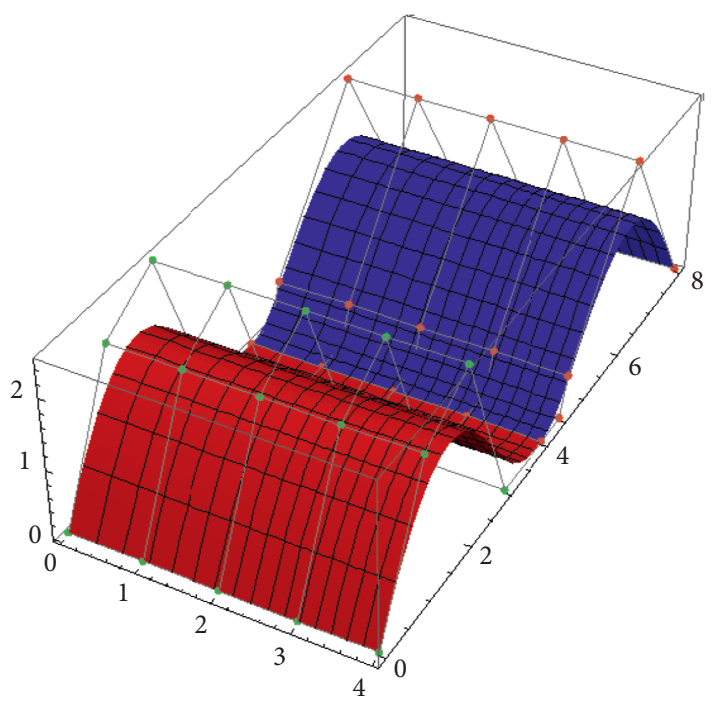

(c)

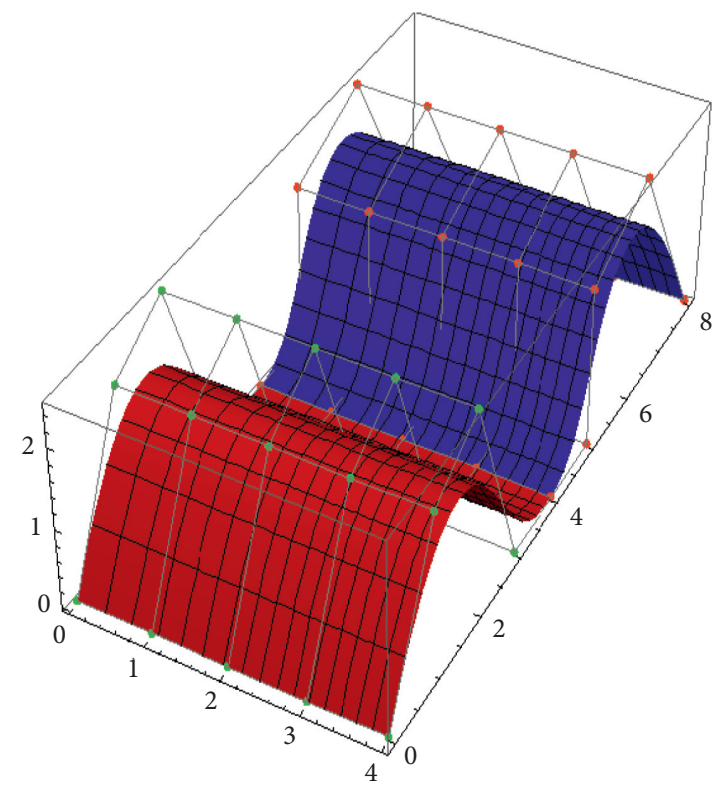

(b)

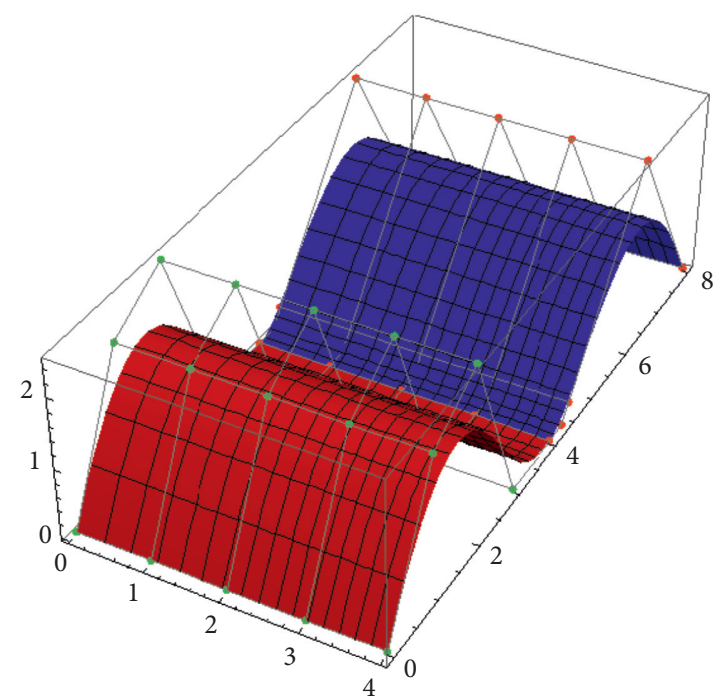

(d)

FiguRE 12: The effect of changing scaling factor on quartic GT-Bézier surfaces. (a) $f=0.35, \alpha, \beta, \alpha^{*}, \beta^{*}=1$. (b) $f=0.5, \alpha, \beta, \alpha^{*}, \beta^{*}=1$. (c) $f=1, \alpha, \beta, \alpha^{*}, \beta^{*}=1$. (d) $f=1.5, \alpha, \beta, \alpha^{*}, \beta^{*}=1$.

decreases), and when $f$ is fixed and the values of shape parameters are increased (or decreased), the surfaces move closer to (away from) the control polygon (control net). This analysis shows that the piecewise GT-Bézier surface is smooth and continuous at the joint, which significantly increases the abilities to solve problems in engineering appearance design by amending the position and shape of surfaces. 


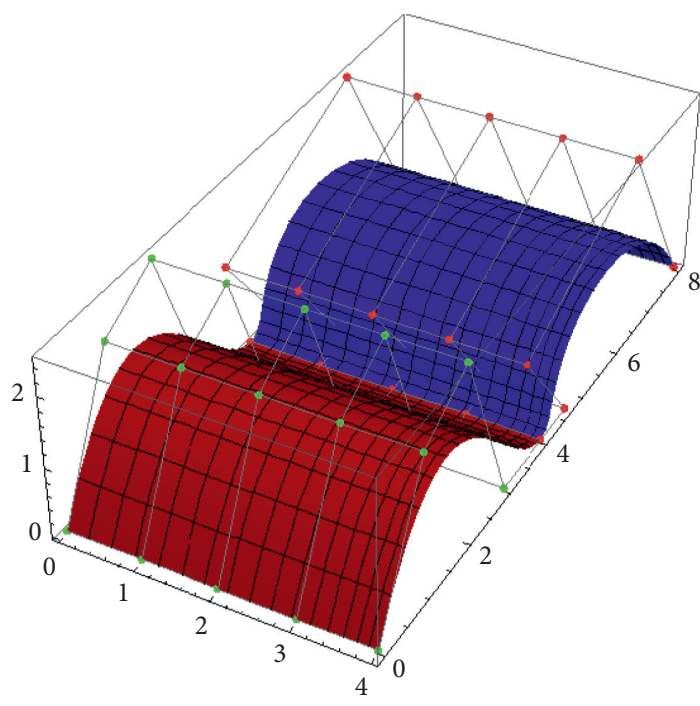

(a)

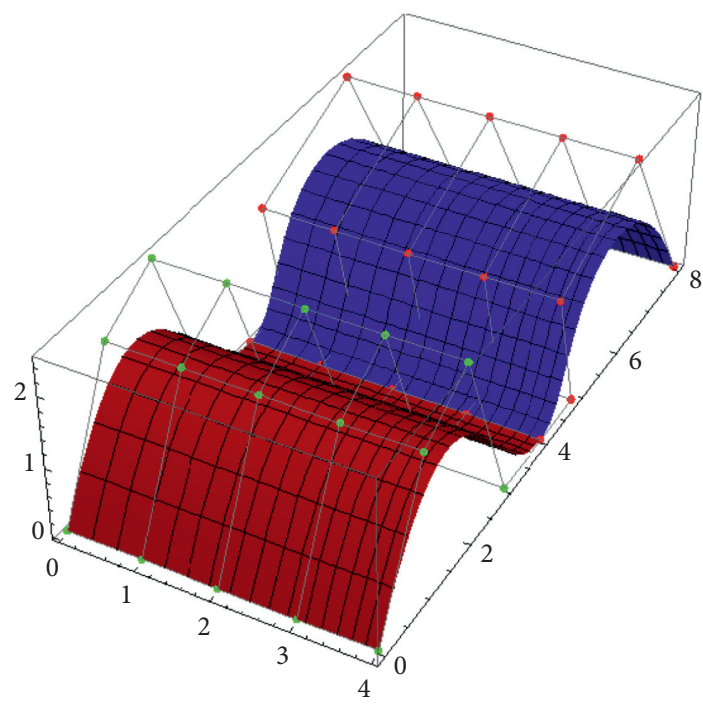

(c)

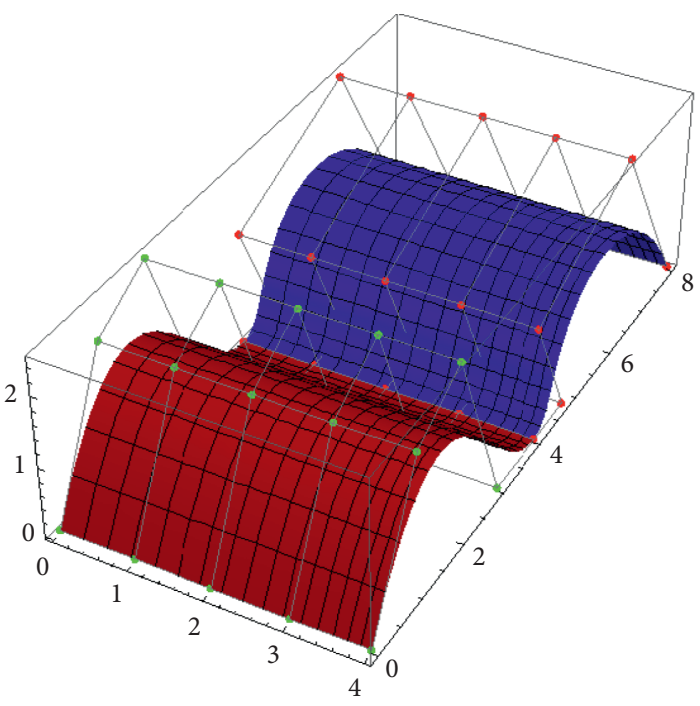

(b)

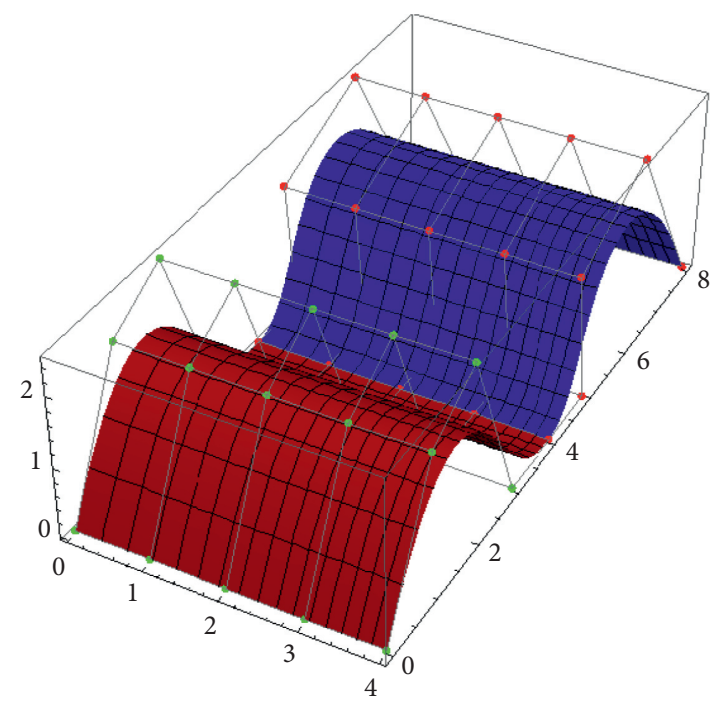

(d)

FigURE 13: The effect of shape parameters on quartic GT-Bézier surfaces. (a) $f=0.5, \alpha, \beta, \alpha^{*}, \beta^{*}=0$. (b) $f=0.5, \alpha, \beta, \alpha^{*}, \beta^{*}=0.25$. (c) $f=0.5, \alpha, \beta, \alpha^{*}, \beta^{*}=1$. (d) $f=0.5, \alpha, \beta, \alpha^{*}, \beta^{*}=0.5$.

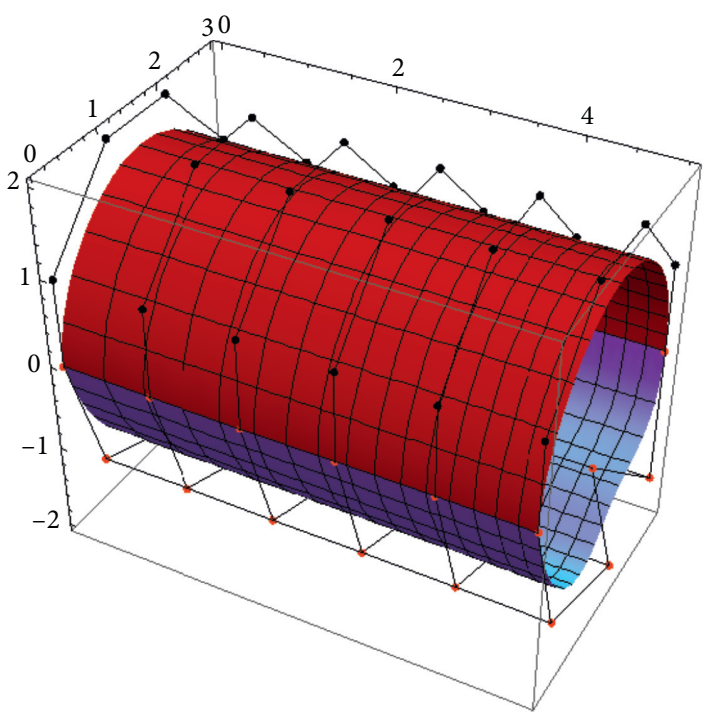

(a)

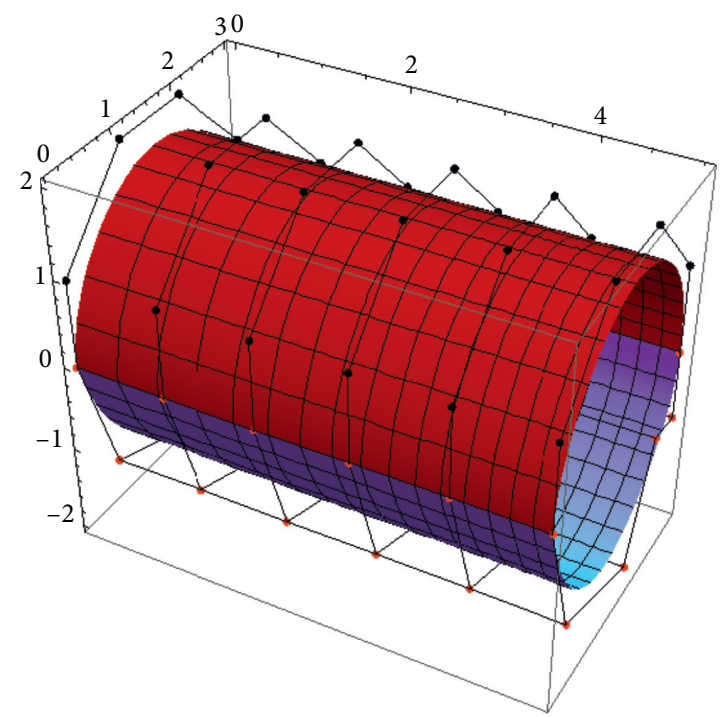

(b)

FIgURE 14: Continued. 


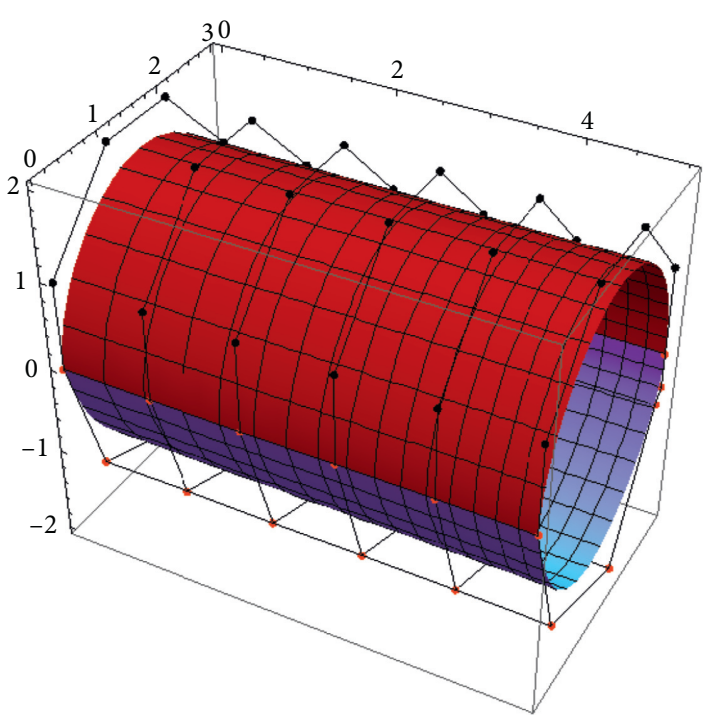

(c)

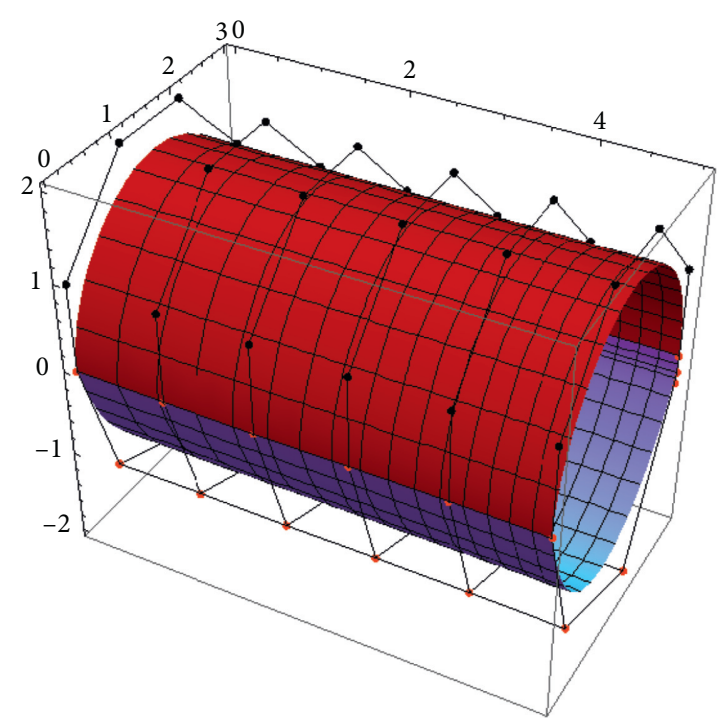

(d)

FIGURE 14: The effect of changing scaling factor on quintic GT-Bézier surfaces. (a) $f=0.25, \alpha, \beta, \alpha^{*}, \beta^{*}=1$. (b) $f=0.5, \alpha, \beta, \alpha^{*}, \beta^{*}=1$. (c) $f=1, \alpha, \beta, \alpha^{*}, \beta^{*}=1$. (d) $f=0.35, \alpha, \alpha^{*}=0, \beta, \beta^{*}=1$.

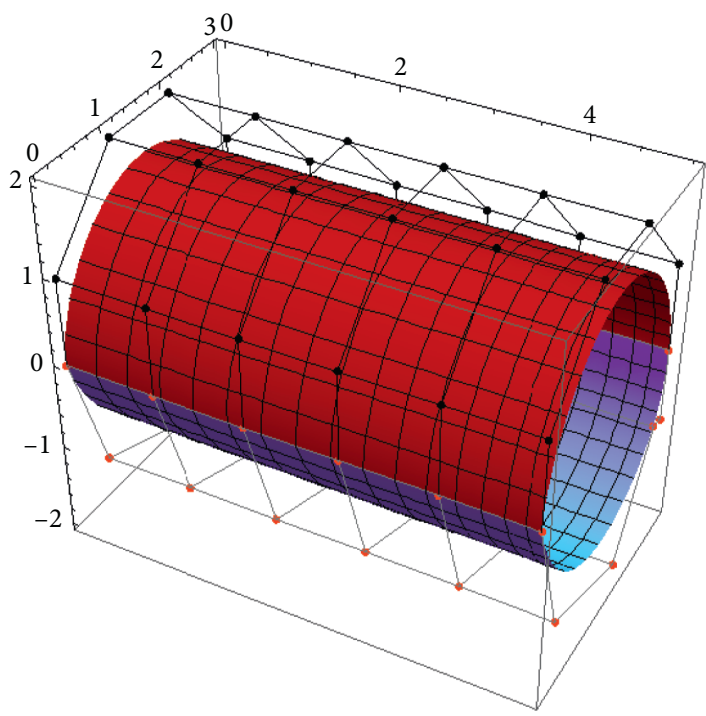

(a)

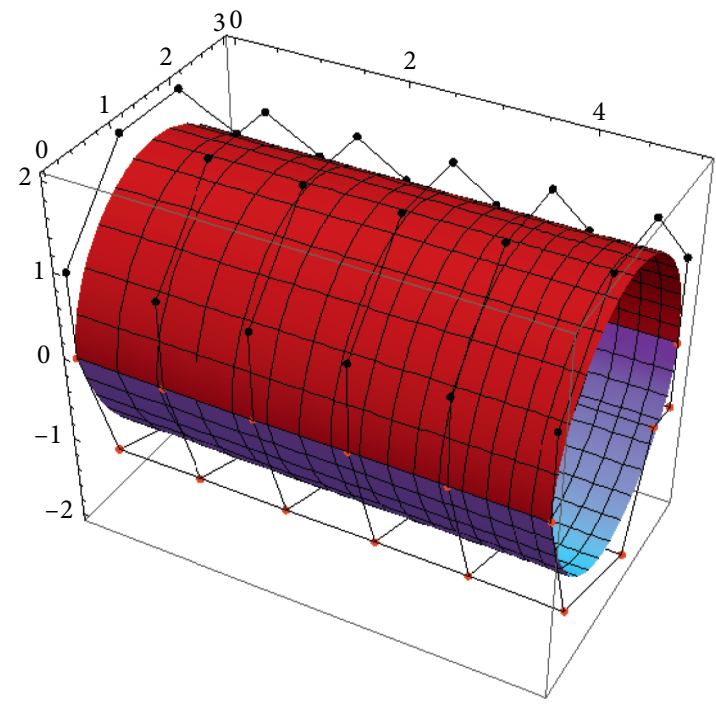

(b)

Figure 15: Continued. 


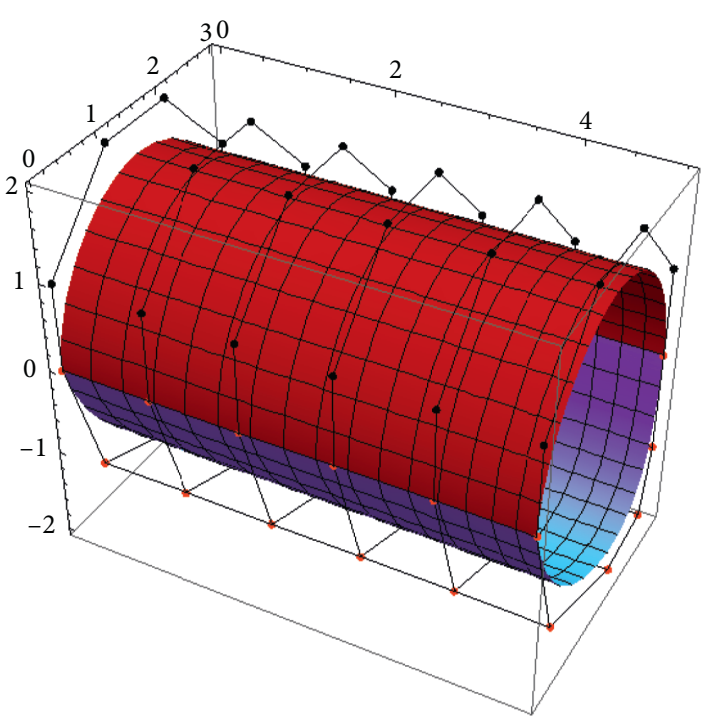

(c)

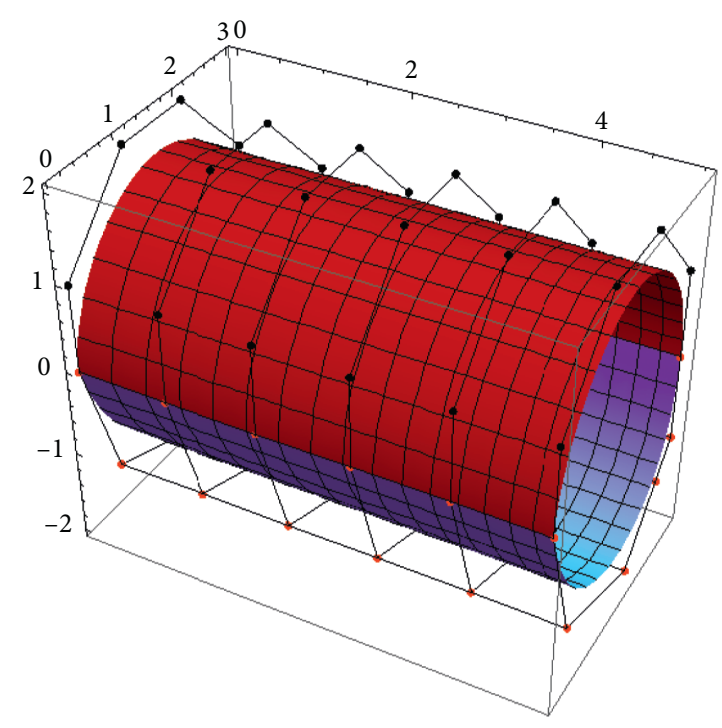

(d)

FIGURE 15: The effect of shape parameters on quintic GT-Bézier surfaces. (a) $f=0.5, \alpha, \beta, \alpha^{*}, \beta^{*}=0$. (b) $f=0.5, \alpha, \beta, \alpha^{*}, \beta^{*}=1$. (c) $f=0.5, \alpha, \alpha^{*}=-0.5, \beta, \beta^{*}=1$. (d) $f=0.5, \alpha, \alpha^{*}=0, \beta, \beta^{*}=1$.

\section{Conclusions}

New $m$ th order GT-Bézier curves along with two shape parameters which share most properties with the $n$th order classical Bézier curve have been presented in this study. GTBézier surfaces of order $m \times n$ have also been proposed by using GT-Bézier curves of order $m$ and $n$. The contour of GT-Bézier curves and surfaces can be transformed not by modifying the control points but it is by varying the values of the shape parameters. Since the GT-Bézier curves and surfaces have been described by using amendable shape parameters, the connection of such curves and surfaces is unwrinkled than that of traditional Bézier curves and surfaces. In contrast with other curve-forming techniques, the technique proposed here can develop curves whose degree and mathematical complication are not increased which make it more useful in practical submissions. Some curve and surface design examples exhibit that this scheme is more convenient, flexible, and effective for both curve and surface interaction modeling and has significant mathematical and applied applications. Additionally, this scheme can be applied to generate trigonometric surfaces over triangles with adjustable shape parameters [37].

\section{Data Availability}

The experimental data used to support the findings of this study are available within this paper.

\section{Disclosure}

The second author presented this paper in C-2018-21st International Conference on Humans and Computers, March 28th and 29th, 2019, at Shizuoka University, Hamamatsu, Japan, as a keynote speaker.

\section{Conflicts of Interest}

The authors declare that there are no conflicts of interest regarding the publication of this paper.

\section{Acknowledgments}

The authors are grateful to the anonymous reviewers for their helpful, valuable comments and suggestions in the improvement of this manuscript. The second author acknowledges the Department of Mechanical Engineering, Shizuoka University, Japan, for providing airfare to attend the C-2018-21st International Conference on Humans and Computers, March 28th and 29th, 2019, at Shizuoka University, Hamamatsu, Japan, as a keynote speaker.

\section{References}

[1] X.-A. Han, Y. Ma, and X. Huang, "A novel generalization of Bézier curve and surface," Journal of Computational and Applied Mathematics, vol. 217, no. 1, pp. 180-193, 2008.

[2] G. Hu, J. Wu, and D. Lv, "Geometric continuity conditions for H-Bézier curves of degree $n$," in Proceedings of the 3rd IEEE International Conference on Image, Vision and Computing, pp. 706-710, IEEE, Chongqing, China, June 2018.

[3] G. Hu, H. Cao, X. Wang, and X. Qin, " $G^{2}$ continuity conditions for generalized Bézier-like surfaces with multiple shape parameters," Journal of Inequalities and Applications, vol. 2017, no. 1, p. 248, 2017.

[4] G. Hu, X. M. Ji, X. Q. Shen, and W. J. Song, "Research on the continuity conditions for CE-Bézier surfaces," Journal of Graphics, vol. 33, no. 5, pp. 62-67, 2012.

[5] G. Hu, X. M. Ji, L. Guo, and W. J. Song, "The quartic generalized C-Bézier surface with multiple shape parameters and continuity condition," Mechanical Science and Technology for Aerospace Engineering, vol. 33, no. 9, pp. 1359-1363, 2014.

[6] J. M. Carnicer, E. Mainar, and J. M. Peña, "A unified framework for cubics and cycloids," in Curve and Surface 
Design, T. Lyche, M. L. Ma-zure, and L. L. Schumaker, Eds., pp. 31-40, Nashboro Press, Brentwood, TN, USA, 2003.

[7] X. Han and S. Liu, "Extension for quadratic Bézier curves," Journal of Zhongnan University, vol. 34, pp. 214-217, 2003, in Chinese.

[8] X. Wu and X. Han, "Extension for cubic Bézier curves," Journal of Engineering Graphics, vol. 6, pp. 98-102, 2005.

[9] X. Wu, X. Han, and S. Luo, "Two different extensions of quartic Bézier curve," Journal of Engineering Graphics, vol. 5, pp. 59-64, 2006.

[10] Z. Liu, “An extension of Bézier curve," Journal of Hefei University of Technology, vol. 27, pp. 976-979, 2004.

[11] X. Han, Y. Ma, and X. Huang, "Shape modification of cubic Quasi-Bézier curve," Journal of Xían Jiaotong University, vol. 41, pp. 903-906, 2007, in Chinese.

[12] X. Qin, G. Hu, and S. Zhang, "New extension of cubic Bézier curve and its applications," Journal of Computer Engineering and Applications, vol. 44, pp. 112-115, 2008.

[13] X. Zhu, Q. Guo, and G. Zhu, "Extension of the quartic Bézier curve with parameters," Journal of Hefei University of Technology, vol. 31, pp. 671-674, 2008, in Chinese.

[14] N. Zhang, X. Qin, G. Hu, and F. Dang, "New extensions of quartic Bézier curve with multiple shape parameters," Journal of Wuhan University of Technology, vol. 31, pp. 156-160, 2009, in Chinese.

[15] L. Yan and J. Liang, "An extension of the Bézier model," Journal of Applied Mathematics and Computation, vol. 218, pp. 2863-2879, 2011.

[16] X. Qin, G. Hu, N. Zhang, X. Shen, and Y. Yang, "A novel extension to the polynomial basis functions describing Bezier curves and surfaces of degree $\mathrm{n}$ with multiple shape parameters," Applied Mathematics and Computation, vol. 223, pp. 1-16, 2013.

[17] G. Hu, G. Wei, and J. Wu, "Shape-adjustable generalized bézier rotation surfaces with multiple shape parameters," Results in Mathematics, vol. 72, no. 3, pp. 1281-1313, 2017.

[18] G. Hu, J. Wu, and X. Qin, "A novel extension of the Bézier model and its applications to surface modeling," Advances in Engineering Software, vol. 125, pp. 27-54, 2018.

[19] B. I. Ksasov and P. Sattayatham, "GB-splines of arbitrary order," Journal of Computational and Applied Mathematics, vol. 104, pp. 63-88, 1999.

[20] J. Zhang and F.-L. Krause, "Extending cubic uniform B-splines by unified trigonometric and hyperbolic basis," Graphical Models, vol. 67, no. 2, pp. 100-119, 2005.

[21] Y. Lü, G. Wang, and X. Yang, "Uniform trigonometric polynomial B-spline curves," Science in China Series F Information Sciences, vol. 45, no. 5, pp. 335-343, 2002.

[22] G. Wang, Q. Chen, and M. Zhou, "NUAT B-spline curves," Computer Aided Geometric Design, vol. 21, no. 2, pp. 193-205, 2004.

[23] X. Han, "Quadratic trigonometric polynomial curves with a shape parameter," Computer Aided Geometric Design, vol. 19, no. 7, pp. 503-512, 2002.

[24] X. Han, "Cubic trigonometric polynomial curves with a shape parameter," Computer Aided Geometric Design, vol. 21, no. 6, pp. 535-548, 2004.

[25] A. Nikolis and I. Seimenis, "Solving dynamical systems with cubic trigonometric splines," Journal of Applied Mathematics E Notes, vol. 5, pp. 116-123, 2005.

[26] E. Dyllong and A. Visioli, "Planning and real-time modifications of a trajectory using spline techniques," Journal of Robotica, vol. 21, pp. 475-482, 2003.
[27] B. Su and L. Zou, "Manipulator trajectory planning based on the algebraic-trigonometric hermite blended interpolation spline," Procedia Engineering, vol. 29, pp. 2093-2097, 2012.

[28] D. G. Schweikert, "An Interpolation curve using a spline in tension," Journal of Mathematics and Physics, vol. 45, no. 1-4, pp. 312-317, 1966.

[29] M.-L. Mazure, "Chebyshev-Bernstein bases," Computer Aided Geometric Design, vol. 16, no. 7, pp. 649-669, 1999.

[30] G. Xu and G.-Z. Wang, "AHT Bézier curves and NUAHT B-spline curves," Journal of Computer Science and Technology, vol. 22, no. 4, pp. 597-607, 2007.

[31] Y. Zhu and Z. Liu, "A class of trigonometric Bernstein-type basis functions with four shape parameters," Mathematical Problems in Engineering, vol. 2019, Article ID 9026187, 16 pages, 2019.

[32] E. Mainer, M. J. Peña, and S. Sánchez-Reyes, "Shape preserving alternatives to the rational Bézier model," Computer Aided Geometric Design, vol. 18, no. 1, pp. 37-60, 2001.

[33] G. Wang and M. Fang, "Unified and extended form of three types of splines," Journal of Computational and Applied Mathematics, vol. 216, no. 2, pp. 498-508, 2008.

[34] T. Bosner and M. Rogina, "Numerically stable algorithm for cycloidal splines," Annali dell'Universita' di Ferrara, vol. 53, no. 2, pp. 189-197, 2007.

[35] P. Costantini, T. Lyche, and C. Manni, "On a class of weak Tchebycheff systems," Numerische Mathematik, vol. 101, no. 2, pp. 333-354, 2005.

[36] E. Mainer and M. J. Peña, "Quadratic-cycloidal curves," Advances in Computational Mathematics, vol. 20, pp. 161-175, 2004.

[37] X. Gang, N. Sun, J. Xu, K.-C. Hui, and G. Wang, "A unified approach to construct generalized B-splines for isogeometric applications," Journal of Systems Science and Complexity, vol. 30, pp. 983-998, 2017.

[38] G. Farin, Curves and Surfaces for CAGD: A Practical Guide, Academic Press, San Diego, CA, USA, 5th edition, 2002. 